\title{
An epidermal patch for the simultaneous monitoring of haemodynamic and metabolic biomarkers
}

\author{
Juliane R. Sempionatto ${ }^{1,2}$, Muyang Lin ${ }^{1,2}$, Lu Yin ${ }^{1,2}$, Ernesto De la paz', Kexin Pei1, \\ Thitaporn Sonsa-ard', Andre N. de Loyola Silva', Ahmed A. Khorshed', Fangyu Zhang ${ }^{\circledR 1}$, \\ Nicholas Tostado', Sheng $X u{ }^{1}{ }^{1 凶}$ and Joseph Wang ${ }^{1}{ }^{1 凶}$
}

\begin{abstract}
Monitoring the effects of daily activities on the physiological responses of the body calls for wearable devices that can simultaneously track metabolic and haemodynamic parameters. Here we describe a non-invasive skin-worn device for the simultaneous monitoring of blood pressure and heart rate via ultrasonic transducers and of multiple biomarkers via electrochemical sensors. We optimized the integrated device so that it provides mechanical resiliency and flexibility while conforming to curved skin surfaces, and to ensure reliable sensing of glucose in interstitial fluid and of lactate, caffeine and alcohol in sweat, without crosstalk between the individual sensors. In human volunteers, the device captured physiological effects of food intake and exercise, in particular the production of glucose after food digestion, the consumption of glucose via glycolysis, and increases in blood pressure and heart rate compensating for oxygen depletion and lactate generation. Continuous and simultaneous acoustic and electrochemical sensing via integrated wearable devices should enrich the understanding of the body's response to daily activities, and could facilitate the early prediction of abnormal physiological changes.
\end{abstract}

ntertwined with concepts of telehealth, the internet of medical things, and precision medicine, wearable sensors offer features to actively and remotely monitor physiological parameters. Wearable sensors can generate data continuously without causing any discomfort ${ }^{1,2}$ or interruptions to daily activity, thus enhancing the self-monitoring compliance of the wearer and improving the quality of patient care ${ }^{2-4}$. Monitoring of single physical parameters, such as the electrocardiogram ${ }^{5-7}$ and blood pressure (BP $)^{8-10}$, as well as biochemical parameters, such as glucose $\mathrm{e}^{11-15}$, using non-invasive wearable sensors has been reported. The importance of integrating multiple sensors on a single device has been demonstrated ${ }^{16}$, where multiple chemical sensors were integrated into a single wrist band. Following this pioneering work, sensor integration is now shifting towards the combination of different sensor modalities. Recent efforts have led to the integration of physical and chemical sensors into a single wearable device-such as combining electrocardiography electrodes with lactate ${ }^{17,18}$ or glucose ${ }^{19}$ sensors to monitor the cardiovascular performance, metabolism, electrolyte balance, or the body temperature of an athlete ${ }^{12,16}$. However, to the best of our knowledge, the in-depth study of the correlation of cardiovascular parameters, particularly $\mathrm{BP}$, with biomarker levels using an integrated hybrid wearable sensor remains unexplored.

Heart rate (HR) and $\mathrm{BP}$, two of the most important vital signs, can dynamically and directly reflect the physiological status of the body. These cardiovascular parameters can be affected by fluctuations of various biomarker concentrations originating from activities such as movement, stress or the intake of food, drinks and drugs that can lead to sudden and sometimes lethal alterations. Parallel BP-chemical sensing could thus have clinical value, especially for people with underlying health conditions-such as the elderly or individuals who are obese, or those affected by diabetes and cardiovascular diseases-as their physiological response to normal day-to-day activities might differ from healthy individuals. Furthermore, the prevention, diagnosis and treatment of many diseases can benefit greatly from simultaneous monitoring of cardiovascular parameters and biomarker levels. These include acute and deadly septic shock, which commonly involves a sudden drop in BP accompanied by rapidly increasing blood-lactate levels ${ }^{20}$, and hypoglycaemia- or hyperglycaemia-induced hypotension or hypertension, which increase the risk of stroke, cardiac diseases, retinopathy and nephropathy in patients with diabetes ${ }^{21-24}$. Tracking of metabolites and haemodynamic parameters using the same device can increase the self-monitoring compliance of a patient, as it simplifies the complex process of using multiple devices for measuring these parameters, thereby circumstantially preventing dangerous cardiac events and saving lives. The combination of transdisciplinary sensing modalities into a single miniaturized skin-conformal wearable device can yield additional advantages. For example, critically ill and premature infants need continuous monitoring of various dangerous conditions, ranging from hypoglycaemia and sepsis-like infection to open-heart surgery, where BP and the level of lactate or glucose need to be monitored continuously ${ }^{25,26}$. The neonate-monitoring systems available at present require the application of multiple, often invasive, sensors coupled to bulky instruments on their tiny bodies that pose severe injury risks and barriers to parent-baby bonding ${ }^{27}$. By integrating different sensing modalities on a single flexible, skin-worn tattoo-like patch, vulnerable patients-from neonates to the elderly-can leverage their monitoring device with minimal discomfort or obtrusiveness. The recent global pandemic has also highlighted the urgent need for remote self-monitoring devices, with particular attention to the management of high $\mathrm{BP}$ and diabetes, which are major factors in the

'Department of Nanoengineering, University of California, San Diego, La Jolla, CA, USA. ${ }^{2}$ These authors contributed equally: Juliane R. Sempionatto,

Muyang Lin, Lu Yin.凶e-mail: shengxu@ucsd.edu; josephwang@ucsd.edu 
death of patients with coronavirus disease 2019 (ref. ${ }^{28}$ ). A comprehensive cardiovascular/biomarker self-monitoring system would enhance the self-awareness of users to their health conditions and alert them as well as their caregivers to the occurrence of abnormal physiological changes.

Here we present a conformal, stretchable and integrated wearable sensor that can monitor BP, HR and the levels of glucose, lactate, caffeine and alcohol towards dynamic and comprehensive health self-monitoring. We use ultrasonic transducers to monitor $\mathrm{BP}$ and HR, and electrochemical sensors to measure the levels of biomarkers. Through strategic material selection, layout design and fabrication engineering, we integrated rigid and soft sensor components, namely customized piezoelectric lead zirconate titanate (PZT) ultrasound transducers and printed polymer composites via an innovative solvent-soldering process into a single wearable conformal sensor with high mechanical resiliency and an absence of sensor crosstalk. Such rational design overcomes engineering challenges related to the integration of different sensing modalities and materials to allow real-time monitoring of cardiovascular parameters and biomarker levels in connection to parallel sampling of the interstitial fluid (ISF) and sweat biofluids. The resulting epidermal hybrid device can emit ultrasonic pulses and sense echoes from arteries while stimulating sweat and extracting ISF through iontophoresis (IP), thereby allowing parallel measurements of BP and HR along with multiple biomarkers in these biofluids. We carried out on-body trials on several human volunteers undergoing various activities and stimuli (exercise and the intake of alcohol, food and caffeine; Fig. 1d). The correlations between metabolic variations and haemodynamic activities under these stimuli were monitored and evaluated. The improved sensor assembly process, leveraging the styrene-ethylene-butylene-styrene block copolymer (SEBS)-based stretchable materials, allowed a fast and reliable fabrication of the stretchable and conformal epidermal sensor for acoustic and electrochemical sensing. Such a device offers comprehensive tracking of the effect of daily activities and stimuli on the physiological status of the user, and enables the collection of previously unavailable data towards understanding the body response to such stimuli while addressing the critical post-pandemic needs for remote telemetric patient monitoring.

The multimodal sensing wearable patch is depicted in Fig. 1a. SEBS was used as the stretchable and conformal substrate to support the electrodes and connections printed with customized inks
(Fig. 1e and Supplementary Fig. 1). The stretchable substrate and inks allow the high conformity, flexibility (Fig. 1f(i)) and stretchability (Fig. 1f(ii)) required for wearable devices. The BP sensor consists of an array of eight piezoelectric transducers that are aligned with the carotid artery on application to the neck to obtain optimal ultrasonic signals (Supplementary Figs. 2-4). During sensing, the piezoelectric transducers were activated with electrical pulses, transmitting ultrasound beams to the artery, and the time of flight of the echoes from the anterior and the posterior walls of the artery was analysed to gauge the dilation and contraction of the arteries (Fig. $1 c, h)$. The optimal BP signal can be selected from the eight transducers with the best alignment to the artery and hence the highest signal quality, thus ensuring reliable BP sensing during movement, when the patch may undergo some displacement (Supplementary Fig. 11). Detailed information regarding the fabrication of the sensor is discussed in Fig. $2 \mathrm{~m}$ and Supplementary Note 1. The characterization of the BP sensor is discussed in Supplementary Note 3 and Supplementary Fig. 10. Chemical sensing was realized through non-invasive sweat stimulation (via transdermal pilocarpine delivery) at the IP anode, along with ISF extraction at the IP cathode. Lactate, alcohol and caffeine were monitored only in sweat, whereas glucose was monitored only in the ISF. Detailed information regarding the monitoring of the ISF and sweat analytes via IP is presented in Supplementary Note 4 (Fig. 1b). Chronoamperometry (CA) was used for the electrochemical detection of the hydrogen peroxide product of the glucose oxidase, lactate oxidase and alcohol oxidase enzymatic reactions, whereas differential pulse voltammetry (DPV) was used for the detection of caffeine. The detailed electrode modifications and reaction mechanisms are discussed in Supplementary Fig. 5. The analytical performance of each chemical sensor is shown in Fig. 1g, Supplementary Figs. 6-9 and Supplementary Note 2.

\section{Results and discussion}

Crosstalk study. The performance of the integrated sensor for multiplexed sensing requires reliable data generation from the individual sensors, with no crosstalk between the two sensing modalities. Here, signal crosstalk between the acoustic and electrochemical transducers was prevented by spatially separating both components and using solid-state ultrasound and sensing hydrogel layers (Supplementary Figs. 26 and 27). The device was designed with an optimal distance between the individual detection compartments to ensure successful acoustic BP and HR sensing, IP extraction and

Fig. 1 | Design and mechanism of the stretchable integrated BP-chemical sensing patch. a, Illustrations of the placement of the sensor and the enzymatic chemical sensors for ISF and sweat. $\mathbf{b}$, Illustration of the acoustic and electrochemical sensing components of the sensor along with hydrogels for sweat stimulation (left) and ISF extraction (right), respectively. c, Acoustic sensing and IP mechanism of the integrated sensor. The transducer applies ultrasound pulses that generate echoes from the anterior and posterior walls of the artery. Chemical sensing starts with the application of an IP current from a positive terminal (anode) to a negative terminal (cathode), which allows the electro-repulsive delivery of a sweat-stimulating molecule $\left(\mathrm{P}^{+}\right.$, pilocarpine nitrate). After pilocarpine delivery, stimulated sweat-containing biomarkers (such as lactate, caffeine and alcohol) are collected and quantified on the left-hand side of the device. The IP current leads to osmotic flow of the biomarkers (such as glucose) from the ISF to the skin surface, allowing its collection and analysis on the right-hand side of the sensor. $\mathbf{d}$, Schematics showing the different daily activities performed by an individual, labelled with their related biomarkers (alcohol, caffeine, lactate and glucose), followed by their effects on the physiological behaviour of an individual (body response). The inputs are transduced and outputted as BP, HR and electrochemical signals by the device, reflecting the physiological status of the body. e, The layer-by-layer layout of the integrated sensor. $\mathbf{f}$, Photos of the sensor under bending (i) and stretching (ii). $\mathbf{g}$, Mechanisms of detection for the electrochemical sensors. (i) Amperometric measurements using enzyme-based sensors. The Prussian-blue (PB) working electrode was modified with the redox enzymes lactate oxidase, glucose oxidase or alcohol oxidase, allowing the biocatalytic oxidation of lactate, glucose or alcohol molecules to pyruvate, gluconic acid or acetaldehyde (products), respectively, along with the production of hydrogen peroxide. The typical electrochemical reduction of the liberated hydrogen peroxide to hydroxyl ions (left) was performed in PBS pH 7.4 by applying a potential of -0.2 V. An increase in negative current is observed by an increase in the concentration of the chemical analyte (right). (ii) Caffeine non-enzymatic measurements. Caffeine was oxidized during the sensing process, which resulted in the production of uric-acid analogue molecules and electrons (left). A carbon electrode modified with multi-walled carbon nanotubes (MWCNT) allowed the pulse-voltammetric detection of caffeine following $30 \mathrm{~s}$ accumulation at $-1.2 \mathrm{~V}$ and scanning between $+0.5 \mathrm{~V}$ and $+1.5 \mathrm{~V}$; potential step, $0.004 \mathrm{~V}$; pulse amplitude, $0.05 \mathrm{~V}$; pulse width, $0.05 \mathrm{~s}$; and scan rate, $0.02 \mathrm{~V} \mathrm{~s}^{-1}$. Following an increase in the concentration of caffeine, an increase in the oxidation signal is observed (right). $\mathbf{h}$, Signal-generation mechanism of the ultrasound transducer (top). The pulsed ultrasound signal from the transducer is reflected from the anterior and posterior walls of the artery and collected by the transducer. Signal processing of the ultrasound signal (bottom). The time of flight of the reflected echo can be converted into BP via established transfer functions. 
electrochemical monitoring. The signal generation of the acoustic sensor relies on high-voltage and high-frequency pulses that may induce signal drift in the chemical sensors, whereas the IP extraction, potentiostatic sensing and potential-sweep sensing may also induce noise in the acoustic signals. As shown in Fig. 2a,d, the BP transducers were located $1 \mathrm{~cm}$ away from the chemical sensors, a distance that was determined to be optimal by assessing the crosstalk between the neighbouring sensors (Supplementary Fig. 26).
Such crosstalk effects between the electrochemical and BP sensors were evaluated by recording the corresponding signals during on-body operations. The BP signals were acquired while the potentiostatic electrochemical input was repeatedly switched on and off to assess the effect of the electrochemical sensing of the anodic (Fig. 2e and Supplementary Fig. 24) and cathodic (Fig. 2b) sensors on the BP signal. Similarly, the effect of the acoustic sensing on both sides of the electrochemical sensing was examined by recording the

a

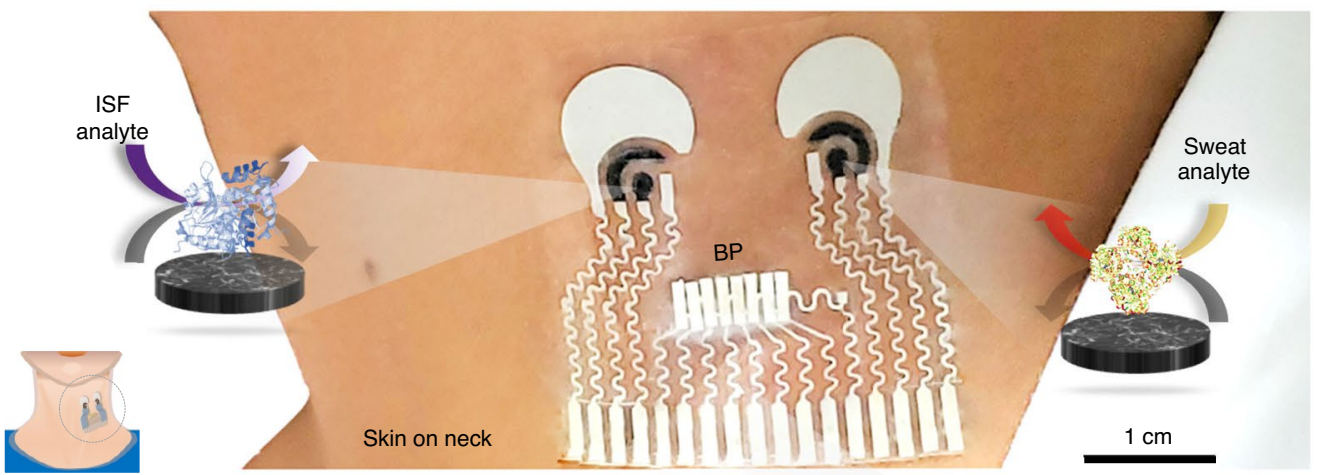

b

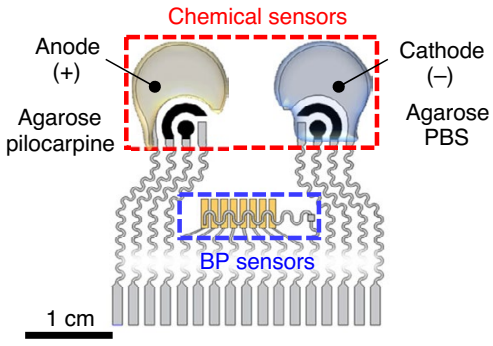

c

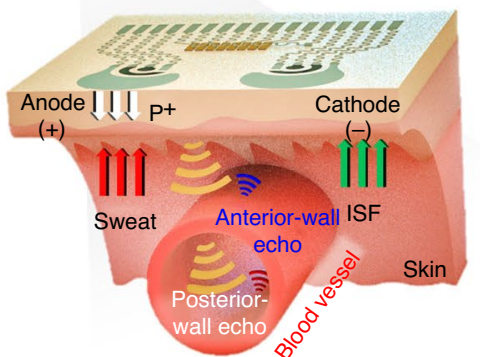

d

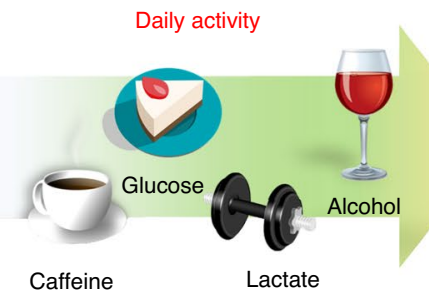

e

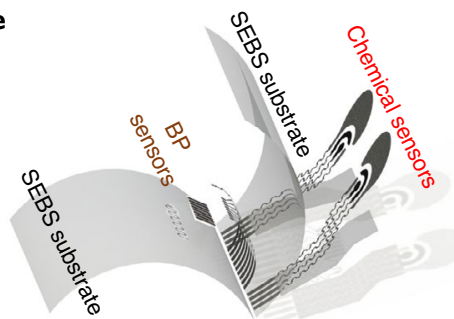

Body response
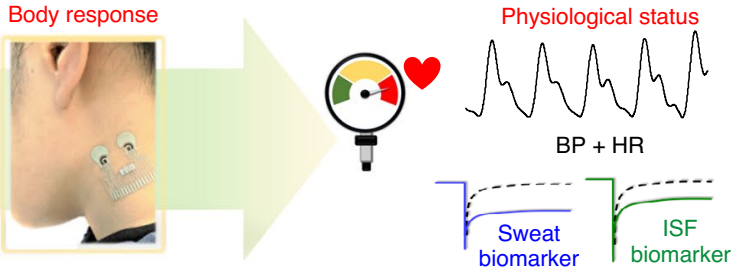

f
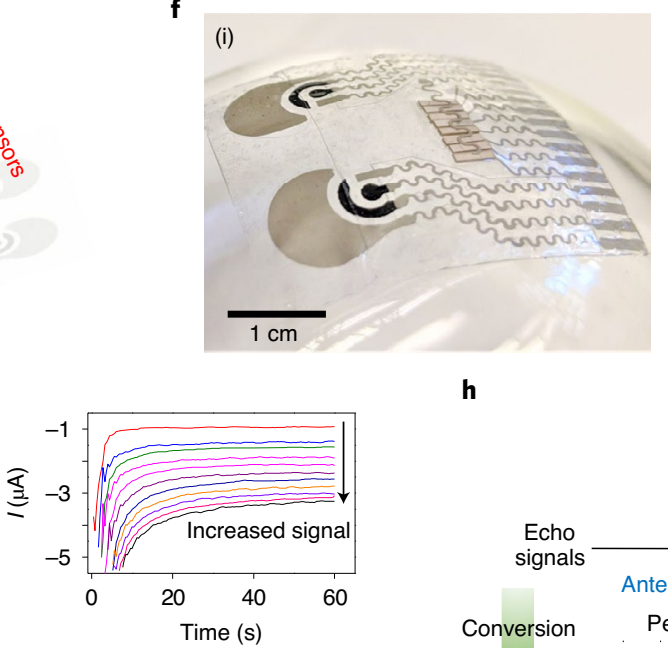

(ii)
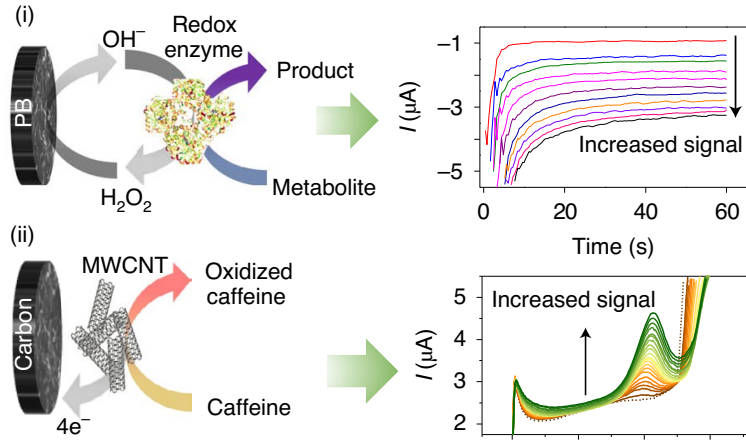

h
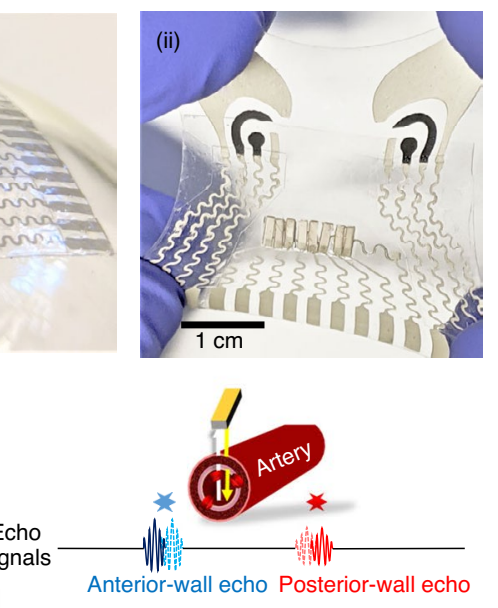

Conversion

Peak shift (artery distension)

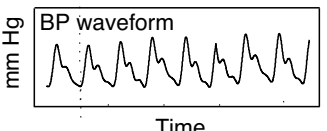


amperometric response while switching the acoustic pulses on and off every 30 s (Fig. 2c,f). Note that without optimization, the electrochemical detection was subject to strong signal interference due to the potential drift caused by the biased voltage from the acoustic pulses (Supplementary Figs. 25 and 26).

Mechanical performance. Mechanical stability is another crucial factor that dictates the reliability of skin-worn sensors when tensile deformations are expected. The impedance of the chemical sensors and the contact resistance to the PZT transducers may vary with the strain applied to the soft conformal device, thereby leading to changes in the measured signals, which affect the reliability of the device. Stability of the PZT contact following mechanical stress was realized by developing a solvent-soldering process based on the fast dissolution and room-temperature curing of SEBS-based materials. During the assembly process, the PZT transducers can be quickly mounted and bonded onto the SEBS substrate and connected to the SEBS-based stretchable silver ink by wetting the electrode surface with toluene (Fig. $2 \mathrm{~m}$ ). The solvent-soldered PZT chips can thus be securely bonded to the printed electrodes without delamination during stretching deformations (Fig. 2n), with substantially improved assembly efficiency in comparison to previous reports ${ }^{10}$. The effects of stretching on the sensing performance were assessed by stretching tests at a uni-axial strain of $20 \%$. The device was stretched repeatedly in vertical (Fig. $2 \mathrm{~g}$ ) and horizontal (Fig. $2 j$ ) directions. The ultrasonic echo signals, against a two-layered Ecoflex, and the current (CA signal) from the bare Prussian-blue electrode (held at $-0.2 \mathrm{~V}$ ), in buffer solution, were recorded after every 200 cycles of stretching at $20 \%$ strain. As shown in Fig. 2h,k, although the intensity of the acoustic transducer signal decreased slightly with stretching, the temporal relationship between each peak that corresponded to two echoes did not change; hence, the deformations did not affect the recorded waveform. Similarly, the electrochemical sensors did not show noticeable current change as the stretching cycle progressed (Fig. 2i,l and Supplementary Figs. $12,13)$. The device also demonstrated good mechanical resilience after it was transferred to the body. Twisting and bending of the sensor on the skin is shown in Fig. 20-q. Mechanical-resilience tests were also performed during active $20 \%$ stretching deformation. The bare and enzyme-modified electrochemical sensors were evaluated in vitro while stretched in two directions (horizontal and vertical), and the BP device was evaluated while capturing the BP signal when turning the neck $90^{\circ}$ to the side; the glucose response did not change during or after 100 stretching cycles, and no changes were observed in the BP waveform while turning the neck (Supplementary Figs. 15-17). Scanning electron microscopy images depicting the structural changes of the surfaces of the printed stretchable silver and carbon traces are in Supplementary Fig. 14, demonstrating that the printed composites are unaffected by the mechanical deformation. A discussion of these images is presented in Supplementary Note 5.
Tracking cardiovascular activities and biomarker levels. The monitoring of both dynamic cardiovascular parameters and biomarker concentrations by the device allows evaluating the effects of common daily activities on the physiological status of an individual and continuously collecting data about their response to these everyday activities. The levels of lactate, glucose, alcohol and caffeine in the body can fluctuate due to common daily activities; furthermore, the impact of these levels on BP also varies based on the physical conditions of the individual. The parallel measurement of biomarkers and BP allows collecting the responses of an individual to such daily activities. The ability of the device to track multiple biomarkers while capturing cardiac parameters can further help deconvolute the additive effects of multiple stimuli on physiological parameters, which has great implications for self-monitoring in personalized health management. To study the effects of each activity on the cardiac parameters, measurements were performed before and after the stimulus. Tests were performed by monitoring $\mathrm{BP}$ along with key sweat and ISF biomarkers, corresponding to specific medical situations. More complex patch designs (illustrated in Supplementary Figs. 34 and 35) can be used for the simultaneous monitoring of BP and multiple sweat chemical markers.

Exercise, comprising any action that demands physical effort, has a major impact on the physiological response of the body, including changes in the HR, BP and levels of lactate. During prolonged exercise, the levels of lactate in the blood and sweat increase due to metabolic stress ${ }^{29}$, the HR is raised to meet the muscle demand for oxygen and the BP surges due to increased availability of vasodilatory mediators such as nitric oxide ${ }^{30}$. To study these effects, several volunteers were asked to perform stationary cycling at a fixed level for $30 \mathrm{~min}$, followed by $5 \mathrm{~min}$ of rest. The artery displacement during cycling was within the device coverage (Supplementary Video 2 ). The BP was recorded while sweating was stimulated before and after the exercise for the lactate measurements, and the obtained BP and lactate-level data were validated using a commercial cuff-style BP monitor and a blood-lactate meter. As expected, large changes in the BP and HR readings were observed after exercise, increasing to $150 \mathrm{~mm} \mathrm{Hg}$ and 98 beats per minute (b.p.m.), respectively (Fig. $3 a(i)$,(ii)). The levels of lactate in sweat also increased, with low lactate levels recorded at the beginning, which increased twofold after the exercise (Fig. 3a(iv),(v)). The BP and sweat-lactate data collected from the device agreed well with the validation methods, as shown in Fig. 3a(iii)-(vi). Note that as no exogenous drugs were used to affect the BP of the volunteers, the relaxation and contraction of the elastine- and collagen-rich central arteries due to exercise could be considered negligible and no additional recalibration of the acoustic sensor was thus needed during the experiment.

As another commonly seen unhealthy stimulus, excessive alcohol consumption has been shown to increase cardiovascular risks via alcohol-induced hypotension and hypertension ${ }^{31,32}$. Alcohol may have different effects on BP, depending on the amount

Fig. 2 | Characterization of the multimodal wearable sensor. a-c, Studies of the signal interference crosstalk between the ISF electrochemical and BP sensors (a), showing the BP-signal recording during the application and removal of the CA detection potential (b) as well as the ISF-sensor signal recording at initiation and pauses in ultrasound-signal generation at intervals of 30 s over 3 min (c). $\mathbf{d}$-f, Studies of the signal interference crosstalk between the sweat electrochemical sensor and BP transducer (d), showing the BP-signal recording during the application and removal of the CA detection potential (e) as well as the sweat-sensor signal recording at the initiation and pauses in ultrasound-signal generation at intervals of 30 s over 3 min (f). g, Photos of the sensor under normal conditions (left) and a $20 \%$ vertical strain (right). h, Envelopes of the raw echo signals before and after every 200 cycles of vertical stretching up to 1,000 cycles. $\mathbf{i}$, Electrochemical response every 200 stretching (vertical) cycles up to 1,000 cycles. j, Photos of the sensor under normal conditions (left) and $20 \%$ horizontal strain (relative standard deviation $=1.09 \%$ ). $\mathbf{k}$, Envelopes of the raw echo signals before and after every 200 cycles of horizontal stretching up to 1,000 cycles. I, Electrochemical response at every 200 stretching (horizontal) cycles up to 1,000 cycles (relative standard deviation $=12.24 \%$ ). $\mathbf{m}$, PZT transfer process. (i) Toluene is drop cast on the electrode pad to dissolve the SEBS trace. (ii) Softened silver ink ready for bonding with the transducer. (iii) Drop cast of toluene on transducers for bonding with the ground wire. (iv) Binding of the ground layer to the reserved electrode channel. $\mathbf{n}$, Adhesion of the PZT transducers to the substrate. Photos of the pristine device under indentation (i), during horizontal stretching (ii) and after transfer of the ground layer (iii). o-q, Skin conformability and mechanical integrity of the device during twisting (o) and bending (p), and after these deformations (q). RF, radiofrequency. 
and frequency of its consumption as well as genetic factors related to resistance or sensitivity to alcohol ${ }^{33,34}$. Variations in BP following alcohol ingestion are related to direct vasodilation, surge in cortisol secretion and reduced insulin sensitivity ${ }^{35}$. For the sensor experiments focusing on alcohol as the stimulus, the BP and sweat-alcohol levels were measured before and $20 \mathrm{~min}$ after drinking $200 \mathrm{ml}$ of an alcoholic beverage (19\% vol; Fig. 3b). A commercial alcohol breathalyser was used for correlation with the blood-alcohol level. As shown in Fig. 3b, alcohol consumption resulted in an increase in the HR (from 69 to 85 b.p.m.) and BP (from 120 to $136 \mathrm{~mm} \mathrm{Hg}$ ) a

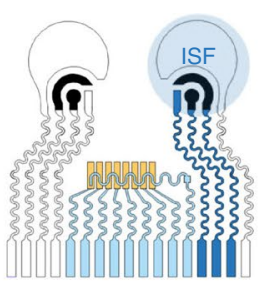

d

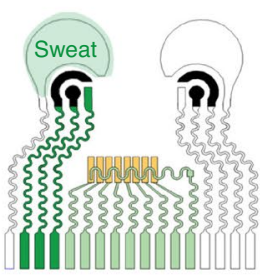

g

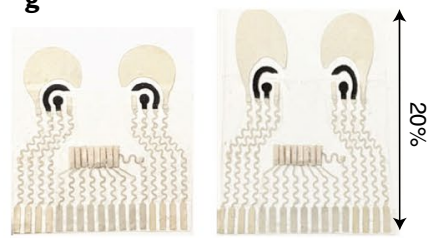

$\overline{0.5} \mathrm{~cm}$

j

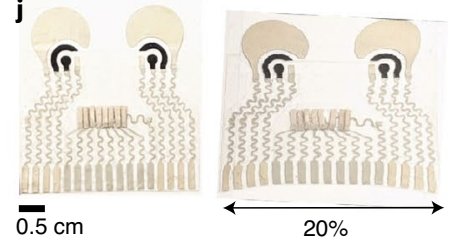

$\mathbf{m}$

m (i)

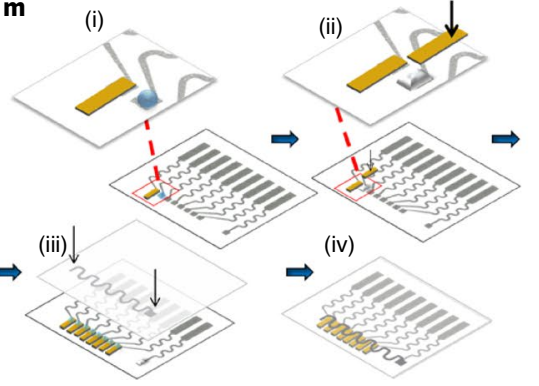

o

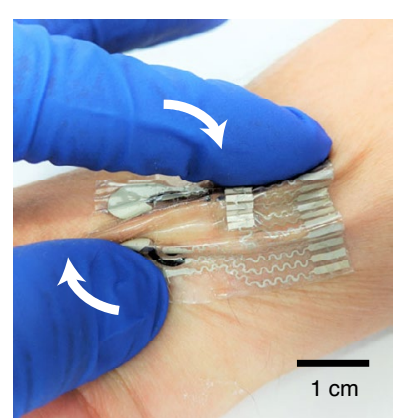

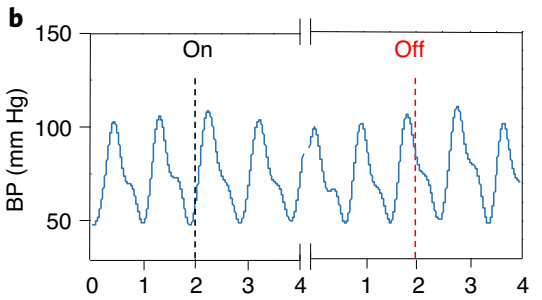

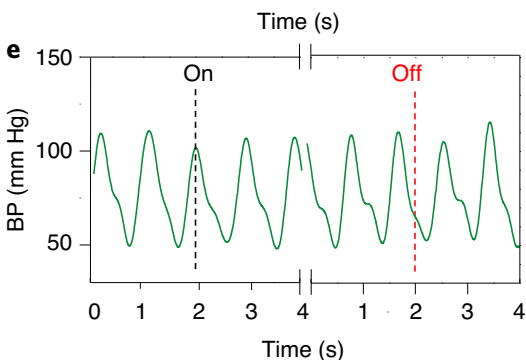

h

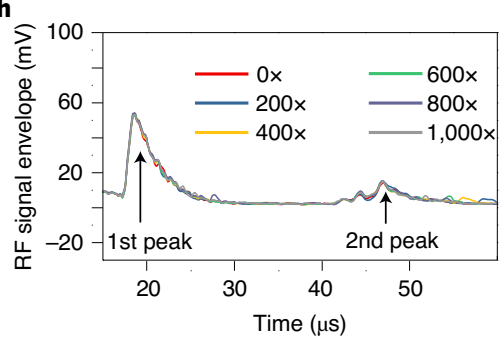

k

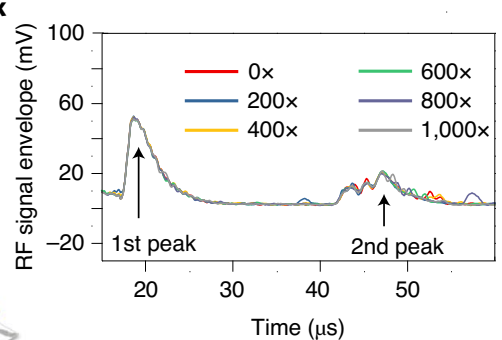

n

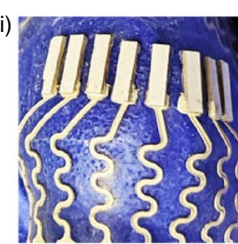

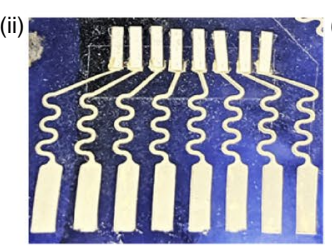

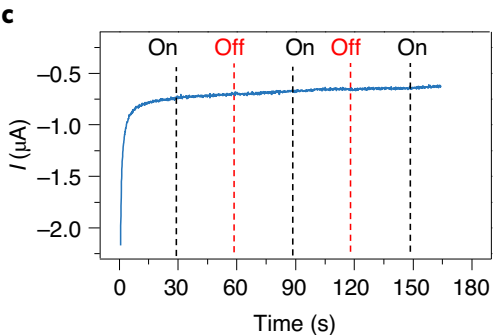

$\mathbf{f}$

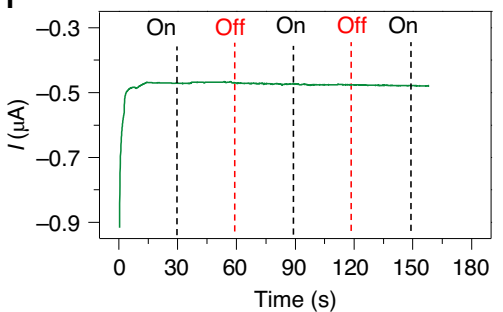

i

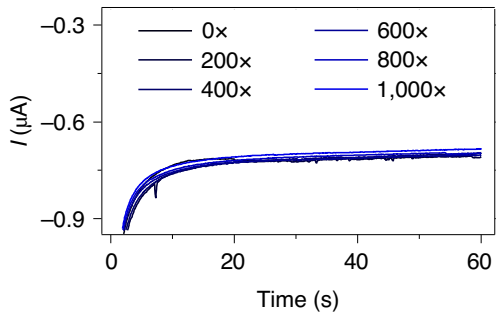

I
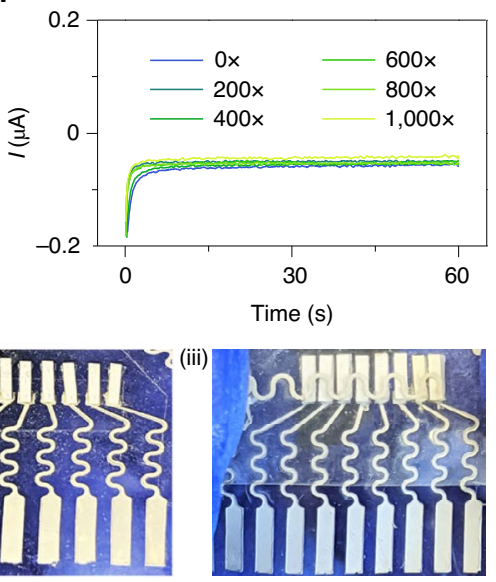

q
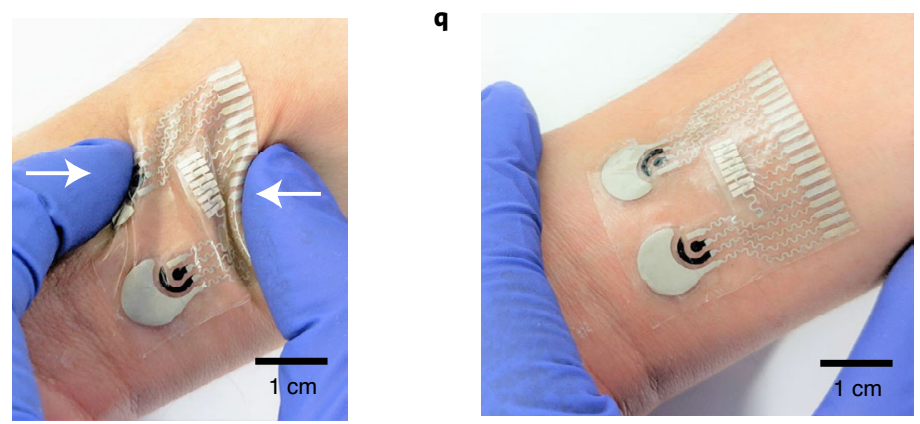

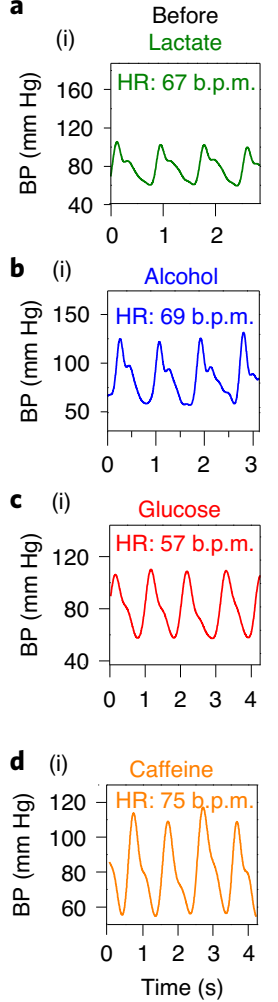
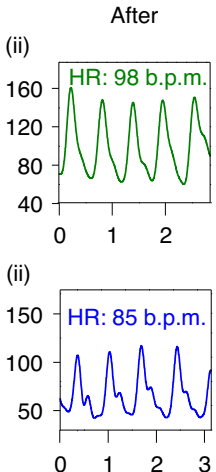

(ii)
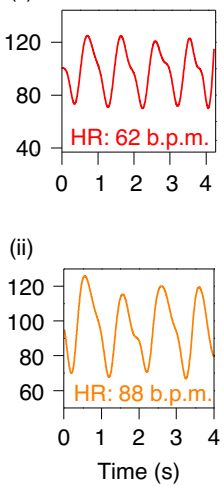
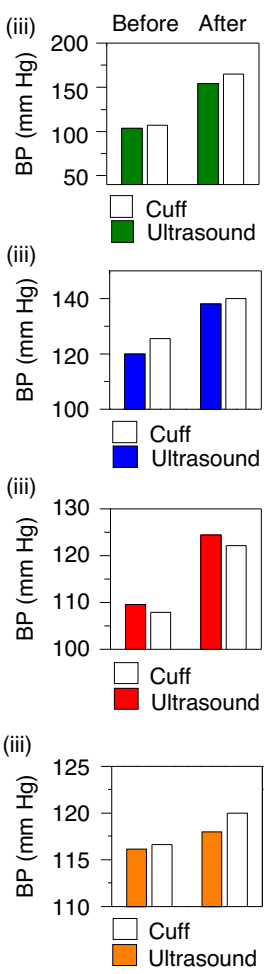
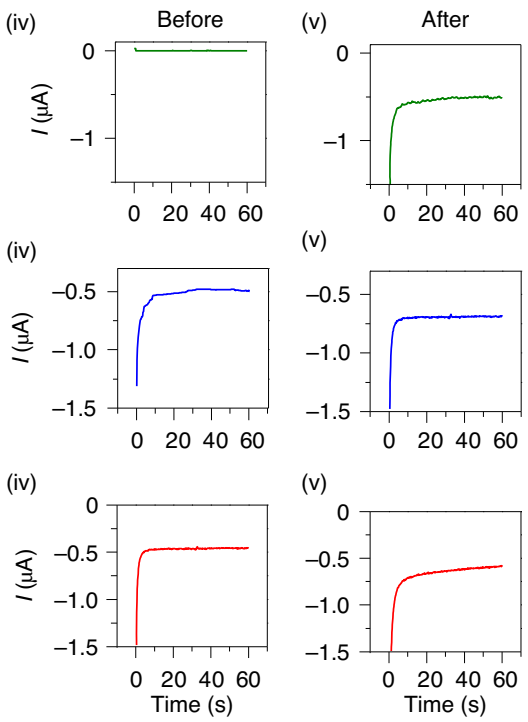

(v)

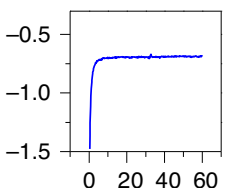

(
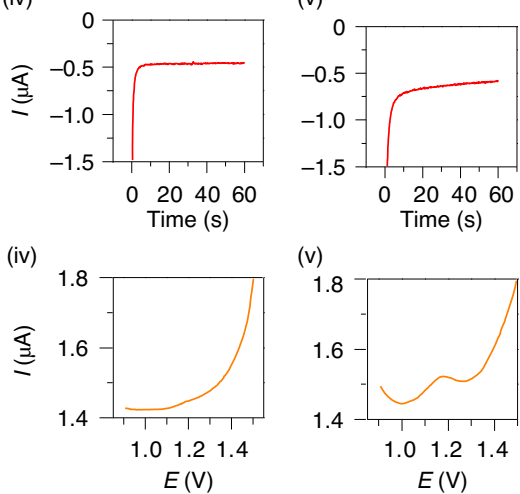

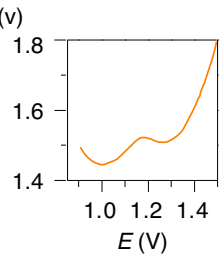

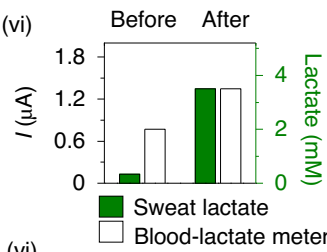

(vi)

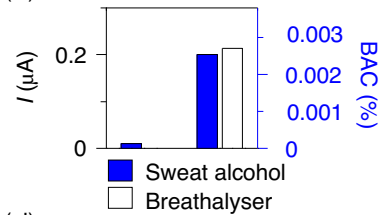

(vi)

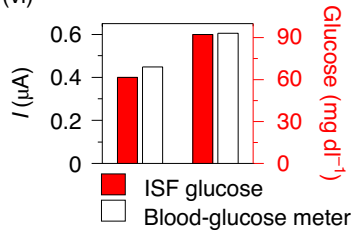

(vi)

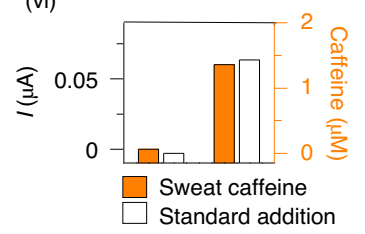

Fig. 3 | On-body evaluation of the hybrid acoustic-electrochemical sensor. a, BP, HR and sweat-lactate studies. (i),(ii), Signal recordings for the BP and HR performance before (i) and after (ii) exercise. (iii), Sensor validation using a commercial cuff and BP readings obtained with the ultrasound transducers. (iv),(v), Signal recordings for sweat lactate before (iv) and after (v) exercise. (vi), Sensor validation using a commercial blood-lactate meter and readings obtained with the electrochemical sensor. b, BP, HR and sweat-alcohol studies. (i), (ii), Signal recordings for BP and HR performance before (i) and after (ii) alcohol intake. (iii), Sensor validation using a commercial cuff and BP readings obtained with the ultrasound transducers. (iv),(v), Signal recordings for sweat alcohol before (iv) and after ( $v$ ) alcohol intake. (vi), Sensor validation using a commercial breathalyser and readings obtained with the electrochemical sensor. BAC, blood alcohol concentration. c, BP, HR and ISF-glucose studies. (i),(ii), Signal recordings for BP and HR performance before (i) and after (ii) food intake. (iii), Sensor validation using a commercial cuff and BP readings obtained with the ultrasound transducers. (iv), (v), Signal recordings for ISF glucose before (iv) and after (v) food intake. (vi), Sensor validation using a commercial blood glucometer and readings obtained with the electrochemical sensor. d, BP, HR and sweat-caffeine studies. (i),(ii), Signal recordings for BP and HR performance before (i) and after (ii) caffeine intake. (iii), Sensor validation using a commercial cuff and BP readings obtained with the ultrasound transducers. (iv),(v), Signal recordings for sweat caffeine before (iv) and after (v) caffeine intake. (vi), Sensor validation through the standard addition method and readings obtained with the voltammetric sensor.

of the volunteer (Fig. 3b(i),(ii)). These results are in agreement with studies showing that intake of a single alcoholic beverage by non-heavy drinkers can lead to a temporary BP spike ${ }^{35}$. Note that for heavy drinkers, there might be a considerable BP morning surge that greatly increases the risk of stroke ${ }^{35}$. Concurrently, the sensor allows the reliable detection of sweat alcohol, as this small polar molecule can be found in sweat with a 1:1 correlation to blood (Fig. $3 \mathrm{~b}(\mathrm{iv}),(\mathrm{v}))^{36}$.

Metabolites such as glucose can also affect the BP waveform by changing the viscosity of blood. Blood viscosity increases under conditions of insulin resistance, altering the flux of blood in the capillaries and hence the shape of the BP pulse $\mathrm{e}^{37,38}$. Studies have shown that individuals with a high $\mathrm{BP}$ are prone to higher levels of blood glucose ${ }^{39}$. To test the effect of the increase in glucose on the $\mathrm{BP}$, healthy individuals without diabetes were asked to consume a sugar-rich meal after fasting. The BP and levels of ISF glucose were recorded using the device before and $15 \mathrm{~min}$ after food consumption, with the glucose level validated using a commercial glucometer at both times. As shown in Fig. 3c(i),(ii), the food-consumption experiment resulted in negligible changes in the $\mathrm{BP}$ and $\mathrm{HR}$, as detected by the sensor. In contrast, the electrochemical biosensor readily detected changes in the levels of ISF glucose after the meal consumption (Fig. 3c(iv),(v)). These data are within the expectation for individuals without diabetes, as glucose-induced BP changes occur only when glucose levels increase to alter the blood pumping through the arteries, which is uncommon for individuals without diabetes, whose glucose is readily regulated by the responsive release of insulin.

Finally, caffeine was chosen as another chemical stimulus commonly used in our daily lives. Caffeine intake is known to lead to an increase in BP through the inhibition of the adenosine receptor and release of stress hormones, such as norepinephrine or cortisol ${ }^{40}$. These biochemical changes can result in transient contractions of the arterial smooth muscle and influence the vascular tone by phosphodiesterase inhibition ${ }^{41}$. The effect of caffeine on BP varies and has been shown to be more pronounced in individuals with hypertension $^{40,41}$. The epidermal BP and caffeine sensor patch was evaluated on individuals with and without caffeine-intake habits, and their BP and sweat-caffeine levels were measured before and $30 \mathrm{~min}$ after the consumption of a caffeine-rich $(80 \mathrm{mg})$ sugar-free energy drink. The amount of caffeine in sweat was validated through a standard addition voltammetric method, spiking caffeine in a collected sweat sample (Supplementary Fig. 19). As illustrated in Supplementary Fig. 29b, the on-body tests on an individual with habitual caffeine intake showed no notable changes in the BP and HR after the consumption of high caffeine doses, reflecting the 
a
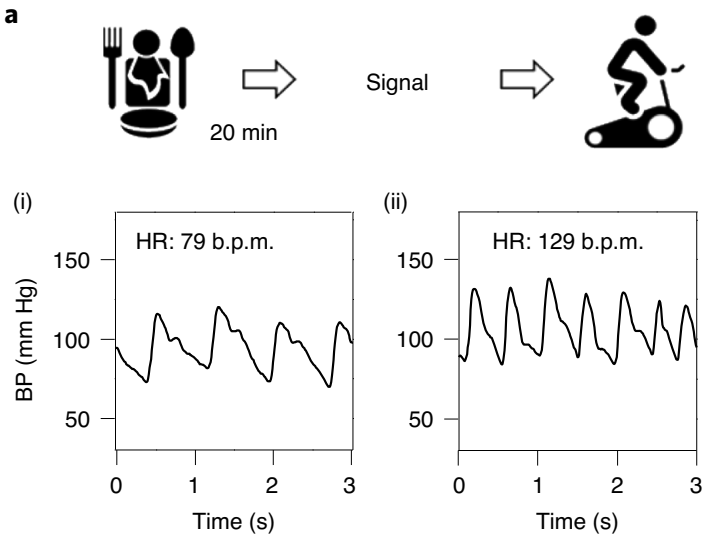

$-1$

20 min

Signal
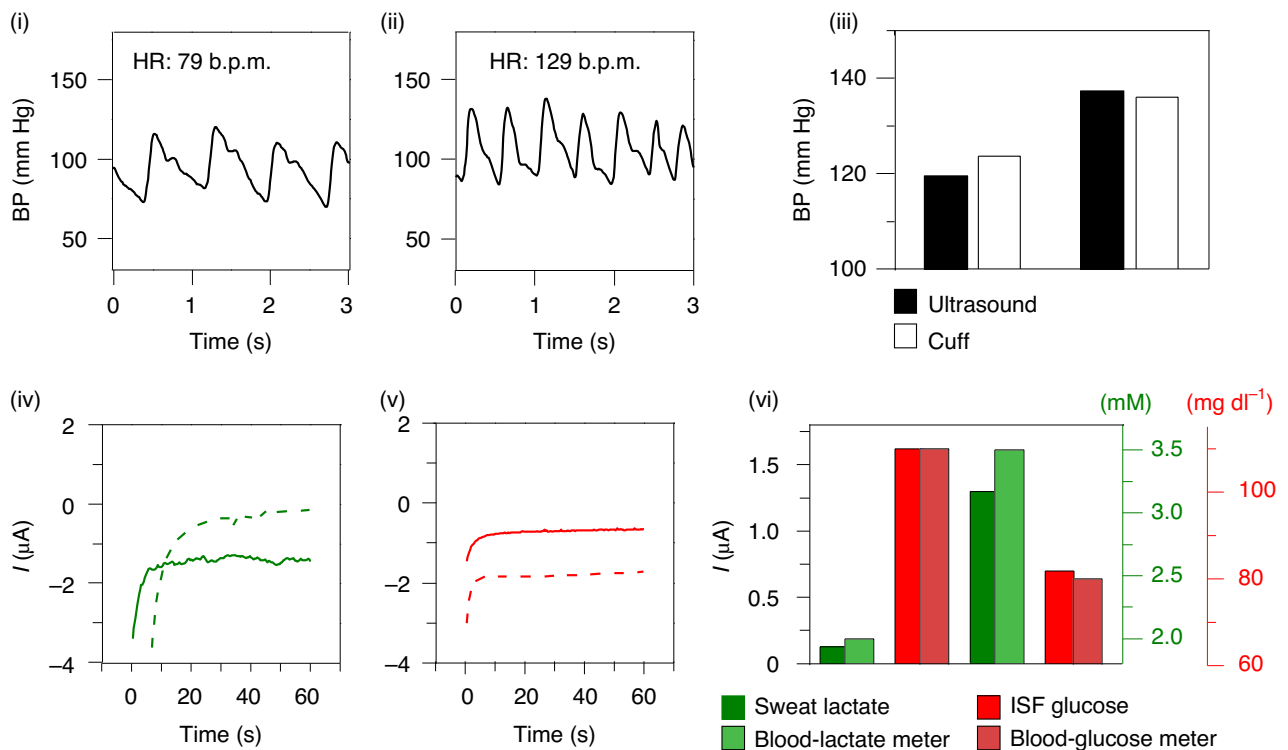

b
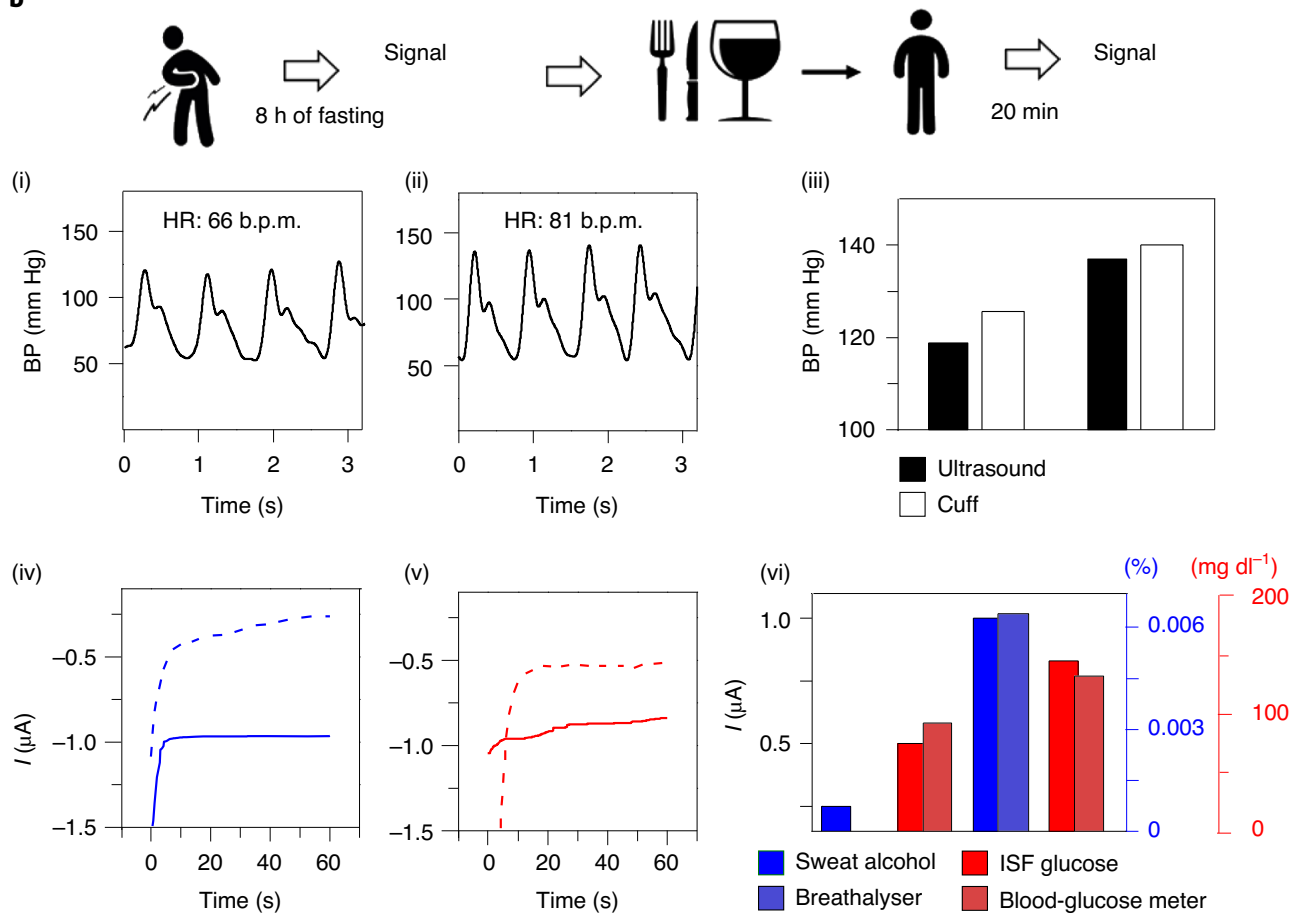

Fig. 4 | On-body evaluation of multiple stimuli. a, Lactate, glucose and BP performance for the illustrated experiment (top). (i),(ii), BP and HR signal recordings before (i) and after (ii) exercise. (iii), Comparison between the BP signal obtained using a commercial cuff and the ultrasound transducer. (iv), Electrochemical sensor signal recordings for sweat lactate before (dashed line) and after exercise (solid line). (v), Electrochemical sensor signal recordings for glucose after having a meal and before exercising (dashed line) and after exercise (solid line). (vi), Comparison between the lactate levels in sweat measured using the electrochemical sensor and a commercial blood-lactate meter as well as the glucose levels in ISF measured using the electrochemical sensor and in blood using a blood-glucose meter. b, Alcohol, glucose and BP performance for the illustrated experiment (top). (i),(ii), BP and HR signal recordings before (i) and after (ii) food and alcohol intake. (iii), Comparison between the BP signal measured using a commercial cuff and the ultrasound transducer. (iv), Electrochemical sensor signal recordings for sweat alcohol before (dashed line) and after (solid line) alcohol intake. (v), Electrochemical sensor signal recording for ISF glucose before (dashed line) and after (solid line) food intake. (vi), Comparison between alcohol levels in sweat measured using the electrochemical sensor and a commercial breathalyser as well as glucose levels in ISF measured using the electrochemical sensor and in blood using a blood-glucose meter. 
caffeine tolerance and healthy BP levels of the volunteer. In contrast, $\mathrm{BP}$ variation was more pronounced for the individual with no habitual caffeine intake (Fig. 3d). The caffeine sensor displayed a flat DPV baseline response before the caffeine intake, whereas the sweat DPV recorded 30 min after the caffeine intake showed a distinct anodic peak current at $1.2 \mathrm{~V}$, corresponding to the caffeine oxidation (Fig. $3 \mathrm{~d}(\mathrm{iv}),(\mathrm{v}))^{42,43}$. The current levels before and after the caffeine intake were compared with the results obtained through the standard addition method for caffeine, demonstrating a good correlation between both parameters (Fig. $3 \mathrm{~d}(\mathrm{vi})$ ). Note that the in vitro electrochemical characterization of the caffeine sensor in $\mathrm{pH} 4.5$ showed current peaks around $1.1 \mathrm{~V}$ (Supplementary Fig. 9). This small potential shift reflects the use of acetate-buffer-loaded agarose gel over the caffeine sensor to minimize the effect of fluctuations in the sweat $\mathrm{pH}$ (that is, $\mathrm{pH} 4.5-7.0$ ).

Device monitoring multiple stimuli. We then evaluated the device in real-life scenarios, where people usually experience multiple activities that may have synergistic or counteracting effects on the physiological responses of the body. The device was evaluated for use in monitoring cardiovascular parameters along with multiple biomarker levels on individuals exposed to multiple stimuli. A common example of a counteracting effect on glucose levels is exercising along with food intake, as glucose can be quickly consumed during exercise to produce energy ${ }^{44}$. Exercise is also expected to increase the BP and lactate levels in an individual, as was shown in previous single-stimuli tests. To study this scenario, the volunteer was asked to consume a sugar-rich meal and then exercise on a stationary bike for $30 \mathrm{~min}$, and the ISF glucose, sweat lactate and BP were monitored before and after each step. As shown in Fig. 4a, normal systolic BP, high glucose $\left(>100 \mathrm{mg} \mathrm{dl}^{-1}\right)$ and low lactate levels were observed before the cycling activity. After the exercise, the glucose levels decreased, accompanied by a considerable increase in the BP, $\mathrm{HR}$ and level of lactate, as predicted from previous tests. Control experiments-performed without any food or exercise-were used to corroborate that the change in signal resulted solely from the increase in the lactate and glucose levels (Supplementary Fig. 32). These findings are in agreement with a previously reported extensive study of a similar wearable electrochemical system ${ }^{15}$. Figure $4 \mathrm{a}$ illustrates that the sensor is able to capture the complex processes resulting from the simultaneous food and exercise stimuli, including the digestion of food to produce glucose as the energy reservoir, the glycolysis reaction consuming the glucose and oxygen to release energy, the increased BP and HR compensating for the oxygen depletion and the lactate generation during the hypoxic condition in exercise.

The influence of the combined intake of alcohol and glucose on $\mathrm{BP}$ and HR, simulating a typical scenario of alcohol consumption during meals, was also studied in volunteering individuals. Based on previous observations, increased glucose levels are not expected to cause large changes to the $\mathrm{BP}$ of the individuals, whereas an increase in BP is expected after the alcohol intake. Therefore, an additive effect is expected on the glucose levels and BP when combining the intake of alcohol and sugary food. Moreover, the digestion of alcoholic drinks, along with the reduced insulin sensitivity caused by alcohol consumption, can further aggravate the increase in glucose levels and $\mathrm{BP}^{15}$. On the other hand, excessive alcohol intake can lead to severe hypoglycaemia and hypotension, even when combined with glucose intake, particularly for insulin-dependent individuals with diabetes ${ }^{45}$. Therefore, parallel monitoring of glucose and BP is important to distinguish between cases of moderate or excessive drinking and prevent drinking-induced accidents, especially for individuals with underlying health conditions ${ }^{46,47}$. Sweat-alcohol, ISF-glucose and BP signals were recorded in the fasting state, after alcohol consumption and after food intake. As shown in Fig. 4b(iv)(vi), before any consumption of food or alcohol, the blood-glucose and -alcohol readings were typical of a non-diabetic fasting-state reading-that is, $90 \mathrm{mg} \mathrm{dl}^{-1}$ glucose and a blood alcohol concentration of $0 \%$-whereas increased BP, glucose and alcohol signals were observed for $20 \mathrm{~min}$ after the stimuli. The observed increase in BP following alcohol intake alone was $16 \mathrm{~mm} \mathrm{Hg}$ (Fig. $3 \mathrm{~b}$ (iii)), rising further to $20 \mathrm{~mm} \mathrm{Hg}$ after the concurrent intake of sugary food (Fig. $4 \mathrm{~b}$ (iii)). Such BP variations demonstrate the synergetic effect of the combining alcohol and glucose intake on the BP. Smaller changes in HR were observed following the alcohol and food intake compared with alcohol intake alone, indicating that different mechanisms were responsible for the increase in BP. Control experiments, carried out without the intake of food or drink, were used to corroborate that the observed signal changes were solely due to the increase in alcohol and glucose (Supplementary Fig. 33), as supported by early findings ${ }^{15}$.

Continuous BP and biomarker monitoring. The ability of the sensor to capture the dynamic biomarker and BP fluctuations during physical activity was also demonstrated. Physically active individuals are expected to have a lower resting BP, considerably reducing the risk of heart-failure events ${ }^{48,49}$. The lower resting BP can further be reflected in a smaller increase in BP during exercise, as physically active individuals signal the body earlier to release nitric oxide to promote enhanced vasodilation ${ }^{48}$. Smaller increases in lactate levels are also expected for active individuals compared with those who are not active ${ }^{50}$. The BP of an individual is expected to decrease following intense exercise activity, eventually returning to its original value, regardless of fitness level ${ }^{51}$. Furthermore, studies have demonstrated a close relationship between the magnitude of the post-exercise decrease in BP and the lactate levels, showing that elevated blood-lactate levels after high-intensity exercise promote larger differences between the pre- and post-exercise BP values ${ }^{52}$. Thus, such complex dynamic processes require the hybrid sensor to operate continuously to capture these real-time fluctuations throughout the activity. Individuals with different fitness levels (physically active and inactive) were asked to perform a 30-min cycling activity at constant intensity while wearing the device (during the whole experiment), and their BP and sweat-lactate levels were monitored continuously until the exercise was stopped. As sweat was generated spontaneously from the activity, IP was not used for this portion of the study. Validation data were also recorded before, $10 \mathrm{~min}$ into and after the exercise. As shown in Fig. 5a (for the physically active individual) and Fig. 5b (for the sedentary individual), considerably higher sweat-lactate levels and increased BP values were observed during the exercise for the sedentary individual compared with the active individual. Higher HR, BP and sweat-lactate levels are expected during exercise for inactive individuals due to the elevated catecholamine levels compared with physically active individuals, thereby leading to differences in BP depending on the fitness levels and cardiovascular system ${ }^{53}$. To address the potential effect of sweat electrolytes on the activity of the Prussian-blue transducer ${ }^{54}$, we used an applied potential that was sufficiently negative $(-0.2 \mathrm{~V})$ to accommodate small possible shifts in the Prussian-blue peak potential (Supplementary Fig. 38). We also demonstrated a patch design capable of performing parallel potentiometric measurements of the sweat-electrolyte levels to correct for the electrolyte effect (Supplementary Fig. 35). The on-body data are shown in Supplementary Fig. 39.

\section{Outlook}

We have shown a conformal skin-worn device capable of parallel monitoring of BP, HR and multiple biomarkers. This has been realized by addressing major engineering challenges in the integration of rigid ultrasound transducers and soft and stretchable electrochemical sensors into a single flexible and stretchable device while 
a

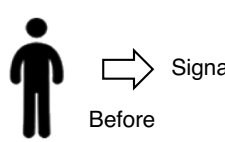

(i)

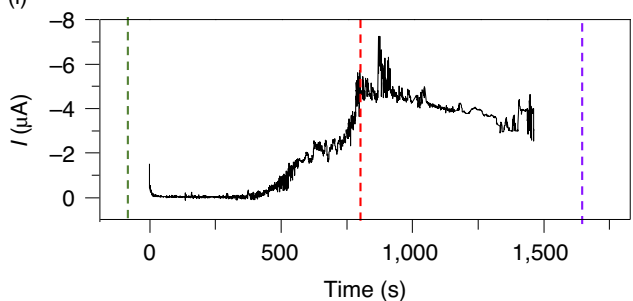

(iii)

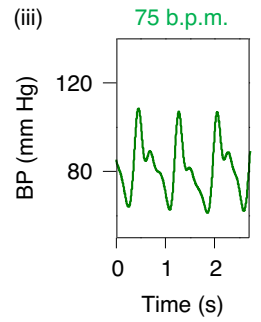

$\begin{array}{lll}\text { (iv) } 109 \text { b.p.m. } & \text { (v) } 85 \text { b.p.m. }\end{array}$
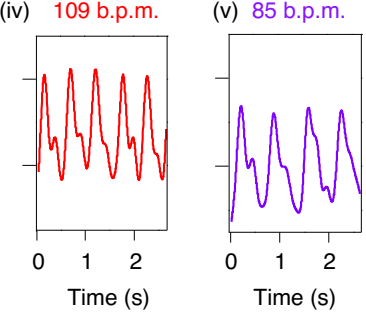

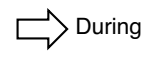

uring

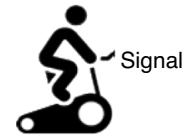

$\Rightarrow \overbrace{\text { After }} \Rightarrow$ Signal
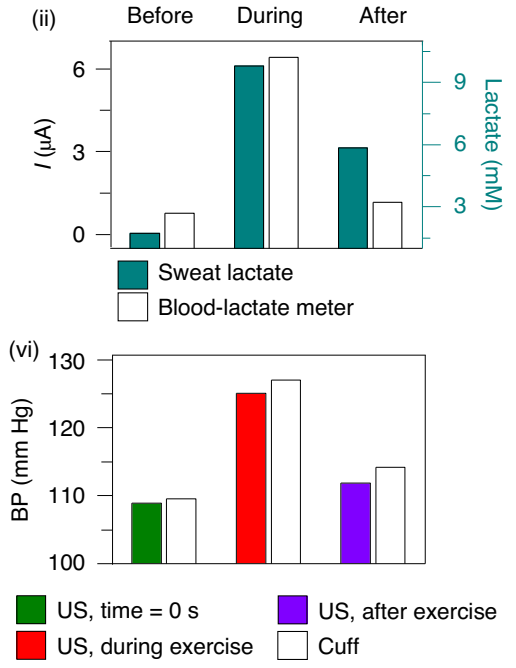

US, during exercise

Cuff

b
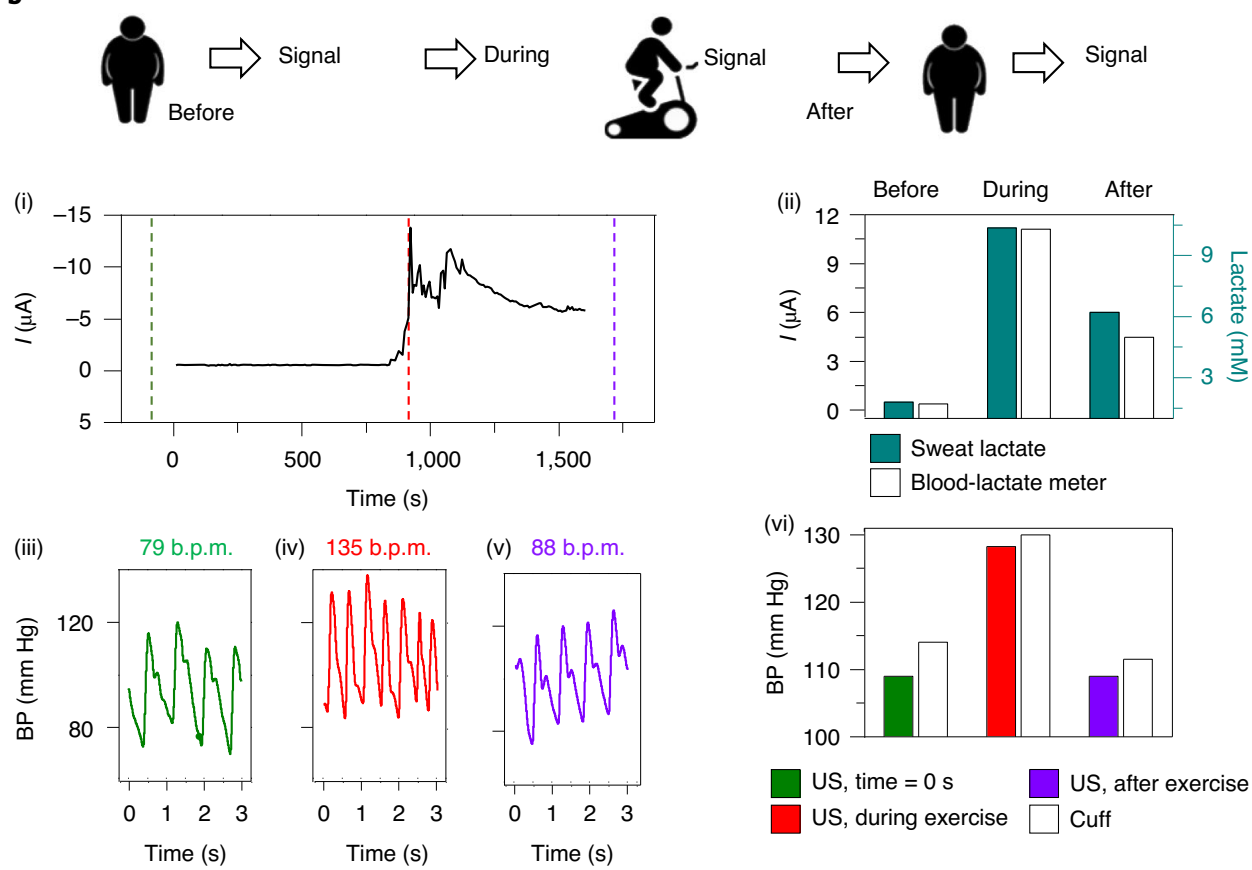

Fig. 5 | Continuous on-body evaluation. a, Continuous lactate, BP and HR performance for an actively fit volunteer during the illustrated experiments (top). (i), Continuous signal recording showing the sweat-lactate profile during stationary cycling. Dashed lines mark the time corresponding to the plotted BP data (before, during and after exericse). (ii), Validation using a commercial blood-lactate meter and electrochemical sensor readings. (iii)-( $v$ ), BP and HR signal recordings before (iii), during (iv) and after (v) stationary cycling. (vi), Comparison between the BP signals before, during and after the exercise obtained using a commercial cuff and ultrasound (US) transducers. b, Continuous lactate, BP and HR performance for the sedentary volunteer during the illustrated experiment (top). (i), Continuous current recording showing the sweat-lactate profile during stationary cycling. Dashed lines mark the time corresponding to the plotted BP data (before, during and after exericse). (ii), Validation using a commercial blood-lactate meter and electrochemical sensor readings. (iii)-(v), BP and HR signal recordings before (iii), during (iv) and after (v) stationary cycling. (vi), Comparison between the BP signal of a commercial cuff and the US transducers before, during and after the exercise activity.

ensuring mechanical performance and avoiding signal crosstalk. The SEBS-based solvent-soldering process has substantially simplified the assembly of a sensor with complex structures, while ensuring reliable mechanical behaviour and continuous epidermal
BP- and biomarker-signal recordings under different chemical and physical stimuli and activities. Signal crosstalk between the acoustic and electrochemical transducers was prevented by spatially separating both components and using solid-state sensing hydrogel layers. 
Repeated mechanical deformation tests demonstrated outstanding durability and reliability of the electrochemical and acoustic sensors.

Such acoustic and electrochemical sensing offers continuous monitoring of the physiological status of the user and its response to multiple everyday activities and stimuli. This multimodal wearable technology has thus been shown to be useful for correlating common daily activities - such as, exercise, drinking and eatingwith changes in BP, HR and biomarker levels. These encouraging results support the possibility of developing hybrid wearable sensors with complex integration of chemical and physical sensors on a single conformal wearable patch for the simultaneous monitoring of multiple relevant parameters. Such integration of reliable and comprehensive epidermal sensors can only be realized with judicious selection of materials, optimized structural engineering and a high-throughput fabrication process in mind. Although the integrated device displays attractive features, there are still opportunities for the following improvements to the measurements of BP, HR and metabolites. (i) The integrated patch relies on the IP pilocarpine stimulation of sweat, which limits the operational use due to the depletion of pilocarpine. Long-term sweat-stimulating drugs (for example, carbachol) could be used to extend the operation period and perform multiple measurements using a single sweat-stimulation step. (ii) Testing of multiplexed patch designs to allow for comprehensive multi-analyte monitoring (Supplementary Fig. 34). (iii) Incorporation of a perspiration-rate sensing interface to normalize the ISF marker readings or use of sweat-suppressive drugs along with an internal standard (sodium) to address potential ISF dilution by passive sweating. (iv) Conducting extensive validations involving a large number of individuals with various health conditions, including patients with diabetes and cardiovascular disease. (v) Full miniaturization of the device through the development of electronics with integrated ultrasound and multi-potentiostatic capabilities, along with signal processing and wireless transmission functionalities. Previous examples have shown the successful miniaturization of wearable devices featuring multiplexed sensing modalities, wireless communications and displays ${ }^{16,55,56}$. The future development of a stand-alone acoustic sensing interface circuit, coupled with artificial intelligence-aided signal processing, will fully transform the present device into a comprehensive skin-worn sensing system. By addressing these opportunities and adding more sensing parameters, we envision a fully integrated multiplexed wearable health-monitoring device that offers insights into the health and physiological status of individuals in the prevention and management of chronic diseases. This device represents a first step towards multimodal wearable sensors that fuse acoustic and electrochemical sensors for more comprehensive monitoring of human physiology and towards telehealth transformation. It thus paves the way into a family of skin-conformal tools capable of providing high-quality and high-density information regarding the status of human health and lays the foundation for next-generation wearable patches capable of hybrid chemical-electrophysiological-physical monitoring.

\section{Methods}

Materials and reagents. Chitosan, acetic acid, bovine serum albumin, L-lactic acid, $\mathrm{NaH}_{2} \mathrm{PO}_{4}, \mathrm{Na}_{2} \mathrm{HPO}_{4}, \mathrm{D}^{+}$-glucose, glucose oxidase from Aspergillus niger type X-S (EC 1.1.3.4), Nafion, agarose, pilocarpine nitrate, Prussian blue (soluble), toluene, ethanol and silver flakes were obtained from Sigma-Aldrich. Graphite powder was purchased from Acros Organics. Lactate oxidase (activity of $101 \mathrm{Umg}^{-1}$ ) was purchased from Toyobo Corp. SEBS (G1645) was received from Kraton Corporation, whereas Ecoflex 00-30 was purchased from Smooth-on Inc. Super-P carbon black was obtained from MTI Corporation. The ultrasound gel pad (AQUAFLEX) was purchased from Parker Laboratories Inc. All reagents were used without further purification.

Sensor fabrication, assembly and electrode modification. The screen-printing was carried out using a semi-automatic MPM-SPM printer (Speedline Technologies) and custom stainless steel stencils developed using AutoCAD software (Autodesk) and produced by Metal Etch Services, with the dimensions $30.48 \mathrm{~cm} \times 30.48 \mathrm{~cm}$ and a thickness of $125 \mu \mathrm{m}$. The electrodes were printed layer-by-layer as illustrated in Supplementary Fig. 1. Bulk PZT was used for the ultrasound transducers, which were diced (Disco Automatic Dicing Saw DAD3220) into rectangular-shaped pixels $(0.8 \mathrm{~mm} \times 3 \mathrm{~mm})$ and sandwiched by two layers of stretchable silver inks as electrodes. The connection between the transducers and the silver traces was realized by adding a toluene droplet to the printed silver traces and placing the transducers on the softened ink. After attaching the PZT transducers, the screen-printed ground connection was placed on the sensor by dissolving the printed traces in a similar fashion. Details of the transducer assembling process are illustrated in Supplementary Fig. 2. The biosensor electrodes were subsequently modified by drop casting the respective enzymes and polymer layers. Details of the ink formulation, printing and assembling processes, and the individual drop-casting protocols for different biosensors are provided in Supplementary Note 1

Sensor in vitro calibration. The fabricated sensors-that is, the lactate, glucose, alcohol and caffeine biosensors-and the PZT acoustic sensors were calibrated separately in in vitro settings. The biosensors were calibrated using $0.1 \mathrm{M}$ PBS (pH 7.4) or 0.01 M acetate buffer ( $\mathrm{pH} 4.5$ ) with successive spiking of the corresponding analytes and recording of the corresponding CA (for lactate, glucose and alcohol) and DPV (for caffeine). Protocols of the in vitro biosensor calibrations are discussed in detail in Supplementary Note 2. The calibration of the BP waveform is discussed in Supplementary Note 3.

Sensor mechanical tests. The mechanical testing was conducted via controlled stretching tests. A programmable motorized linear stage (X-LRQ, Zaber Technologies Inc.) was used to stretch the device with controlled strain and speed. One of the edges of the printed device was taped at the fixed end of the stage and the other to the moving end of the stage. The device was first stretched to $120 \%$ of its original length in the horizontal direction at a speed of $3 \mathrm{~mm} \mathrm{~s}^{-1}$ and then released back to its original size at the same speed. This process was programmed to be repeated 200 times so that the device could be taken from the stage for measurements before remounting for subsequent stretching. The process was repeated until 1,000 cycles of stretching were completed (Supplementary Figs. 12 and 13). This process was repeated for deformations in the vertical direction using the same device. Electrochemical tests under constant deformation of $120 \%$ stretching (horizontal and vertical directions) were conducted after every 200 stretching cycles until 1,000 stretching deformations had been performed (Supplementary Figs. 15 and 16). The resiliency of the sensor was visually inspected by attaching the device to the skin and subjecting it to various deformations (Fig. 2); the corresponding printed surfaces were also characterized via scanning electron microscopy imaging before and after repeated stretching (FEI Quanta 250), as shown in Supplementary Fig. 14.

Sensor crosstalk tests. The crosstalk between the acoustic and electrochemical signals was analysed on-body by monitoring the changes in one signal while the other signal was generated intermittently. For analysing the co-sensor interference from the CA electrochemical measurement to the BP waveform, the BP signals were recorded continuously for at least $4 \mathrm{~s}$ in two stages: when the CA was already being performed by applying a potential of $-0.2 \mathrm{~V}$ to the electrochemical sensors and when the detection potential was turned on after the BP recording had already started. The interference tests from the BP sensor on the CA measurements were performed in the same fashion for the anodic and cathodic sensors as follows: for analysing the crosstalk effect of the acoustic signal generation following the acquisition of the electrochemical signal, the CA signal was recorded continuously for $180 \mathrm{~s}$ while the electric pulses for the BP measurements were delivered to the PZT transducer in an off-on-off-on pattern with a period of $30 \mathrm{~s}$ for each phase. The crosstalk from the DPV and the acoustic signal was evaluated in the same fashion as for the CA tests as follows. The effect of the acoustic signal on the caffeine sensor was evaluated in two stages: first, by recording the DPV signal while the BP recording was being applied, followed by terminating the BP signal when the DPV reached the peak potential and by recording the DPV signal before initiating the BP acquisition at peak potential. The corresponding data obtained are included in Fig. 2 and Supplementary Fig. 24. All signal generation and data acquisition were performed using a $\mu$ Autolab III electrochemical analyser (Metrohm) for the chemical sensors and the 5077PR pulser-receiver (Olympus) for the acoustic sensors. The potentiostat was configurated with a $\pm 5 \mathrm{~V}$ voltage and $1 \mathrm{~mA}$ current limit to avoid overcurrent or overvoltage. The device was visually inspected to ensure that the transducers were fully covered by the SEBS substrates for insulation. No capacitive coupling, short-circuiting or breakdown conduction were observed during the experiment.

Sensor on-body test protocols. Epidermal evaluation of the device was performed on healthy consenting individuals with no previous history of heart conditions, diabetes or chronic pain, and in strict compliance with the protocol approved by the Institutional review board at the University of California, San Diego. The device was placed on the neck of the volunteers for all on-body evaluations. The glucose, lactate, alcohol and BP signals were validated with a commercial glucometer 
(ACCU-CHEK), blood-lactate meter (NOVA Biomedical), breathalyser (BACtrack S80 Pro) and FDA-approved BP cuff (LOVIA), respectively, before every set of measurements using the integrated sensor. Caffeine concentrations were estimated by standard addition methodology using collected sweat (Supplementary Fig. 19). Sweat stimulation and ISF extraction were realized simultaneously using a $\mu$ Autolab III electrochemical analyser to apply a current density of $0.3 \mathrm{~mA} \mathrm{~cm}^{-2}$ between the cathode and anode electrodes for $10 \mathrm{~min}$. A pre-conditioning step was carried out on the skin before sweat generation by applying the same current density using agarose gels in the cathode and anode compartments for $10 \mathrm{~min}$, followed by immediate placement of the device with pilocarpine delivery gel on the conditioned area. Before placing the sensor, the skin was thoroughly cleaned with soap and alcohol wipes. The patch was transferred to the skin using double-sided clean laser tattoo transfer adhesive (Papilio, TM). Openings were made in the adhesive film to expose the sensors and IP electrodes to the skin. For all measurements, a single device was used for each volunteer to perform the 'before' and 'after' tests. The device was kept on the neck of the volunteer throughout the entire experiment, unless otherwise specified. Details of the hydrogel fabrication and skin transfer processes are illustrated in Supplementary Figs. 21-23 and Supplementary Video 1.

The on-body results were acquired using a benchtop CHI 1230A electrochemica analyser for the biosensors and 5077PR pulser-receiver (Olympus) for the acoustic sensors. Food intake refers to the intake of sugar-rich food ( $100 \mathrm{~g}$ cheesecake; $350 \mathrm{kcal}, 22 \mathrm{~g}$ sugar$)$. Alcohol intake refers to the intake of alcohol $(200 \mathrm{ml}$ wine; alcohol, $19 \%$ vol). Caffeine intake refers to the intake of a sugar-free caffeinated drink ( $248 \mathrm{ml} ; 80 \mathrm{mg}$ caffeine). Exercise refers to a 30 -min exercise session on a stationary bike with constant intensity followed by a 5 -min cooling period.

Exercise. Lactate and BP signals were acquired for three healthy volunteers before and after exercise. The device was removed from their skin during the 30-min exercise on a stationary bike and kept in a wet chamber at room temperature. After the exercise, and following a 5-min cooling period, the neck of the volunteer was cleaned with soap and alcohol pads for replacing the same sensor to the same area. The optimal BP signal after the sensor replacement was selected by testing the PZT array in the BP sensor. The influence of device removal or replacement on the signal was studied, as illustrated in Supplementary Fig. 11.

Alcohol intake. Alcohol levels and BP were measured before and 20 min after alcohol consumption. The device was kept on the neck of the volunteer during the entire experiment.

Food intake. Signals for BP and ISF glucose were acquired for three healthy volunteers in the fasting state $(16 \mathrm{~h})$ and $15 \mathrm{~min}$ after consuming the sugar-rich food. The device was kept on the neck of the volunteer throughout the entire experiment.

Caffeine intake. The caffeine levels in the sweat of the volunteer were monitored before and $30 \mathrm{~min}$ after consuming a sugar-free caffeine drink. The device was kept on the neck of the volunteer throughout the entire experiment. For the on-body tests, an agarose gel loaded with acetate buffer $\mathrm{pH} 4.5$ was used, covering only the caffeine sensor. Stimulated sweat was collected before the caffeine ingestion for the standard addition caffeine determination.

Simultaneous alcohol and food intake. The dual modality of the sensor was tested by combining alcohol and food intake. The BP, ISF-glucose and sweat-alcohol levels were measured before and $20 \mathrm{~min}$ after the simultaneous consumption of an alcoholic beverage and the sugar-rich food. The device was kept on the neck of the volunteer throughout the entire experiment.

Food intake and exercise. The dual modality of the sensor was tested for the monitoring of BP as well as the levels of glucose and lactate. The volunteer was first asked to consume a sugar-rich food. Fifteen minutes after the food consumption, ISF glucose, sweat lactate and BP were measured. Next, the device was removed from the volunteer and kept in a wet chamber at room temperature, and the volunteer was asked to perform the physical exercise on a stationary bicycle for $30 \mathrm{~min}$, followed by cooldown for $5 \mathrm{~min}$. After the cooldown interval, the skin was cleaned and the same sensor was used for subsequent measurements of the ISF-glucose, sweat-lactate and BP levels.

Continuous lactate and BP sensing during exercise. The sensor was further tested by monitoring dynamic changes in BP and sweat lactate during continuous physical activity. Individuals with different fitness levels (physically active and inactive) were asked to perform 30 min of cycling activity at constant intensity while wearing the sensor. As sweat was generated spontaneously from the activity, IP and the IP gels were not used for this portion of the study. The BP and blood lactate were measured just before the start of the exercise and the initial sweat-lactate level was measured $5 \mathrm{~min}$ after starting the exercise when sweat was first generated. The BP and blood-lactate signals were recorded again within approximately $10 \mathrm{~min}$. The $\mathrm{BP}$ and blood-lactate levels were also recorded on completion of the exercise for validation.
Reporting Summary. Further information on research design is available in the Nature Research Reporting Summary linked to this article.

\section{Data availability}

All the data generated and analysed during the study are included in the paper and its Supplementary Information.

\section{Code availability}

The LabView code used for processing the transducer raw signals into blood-pressure waveforms is available at https://doi.org/10.5281/zenodo.4433220.

Received: 7 July 2020; Accepted: 12 January 2021; Published online: 15 February 2021

\section{References}

1. Kim, J., Campbell, A. S., de Ávila, B. E.-F. \& Wang, J. Wearable biosensors for healthcare monitoring. Nat. Biotechnol. 37, 389-406 (2019).

2. Yu, Y., Nyein, H. Y. Y., Gao, W. \& Javey, A. Flexible electrochemical bioelectronics: the rise of in situ bioanalysis. Adv. Mater. 32, 1902083 (2020).

3. Khan, Y., Ostfeld, A. E., Lochner, C. M., Pierre, A. \& Arias, A. C. Monitoring of vital signs with flexible and wearable medical devices. Adv. Mater. 28, 4373-4395 (2016)

4. Wang, C., Wang, C., Huang, Z. \& Xu, S. Materials and structures toward soft electronics. Adv. Mater. 30, 1801368 (2018).

5. Lee, S. P. et al. Highly flexible, wearable, and disposable cardiac biosensors for remote and ambulatory monitoring. npj Digit. Med. 1, 2 (2018).

6. Lee, Y.-D. \& Chung, W.-Y. Wireless sensor network based wearable smart shirt for ubiquitous health and activity monitoring. Sens. Actuators B 140, 390-395 (2009).

7. Yapici, M. K., Alkhidir, T., Samad, Y. A. \& Liao, K. Graphene-clad textile electrodes for electrocardiogram monitoring. Sens. Actuators B 221, 1469-1474 (2015).

8. Luo, N. et al. Flexible piezoresistive sensor patch enabling ultralow power cuffless blood pressure measurement. Adv. Funct. Mater. 26, 1178-1187 (2016).

9. Dagdeviren, C. et al. Conformable amplified lead zirconate titanate sensors with enhanced piezoelectric response for cutaneous pressure monitoring. Nat. Commun. 5, 4496 (2014).

10. Wang, C. et al. Monitoring of the central blood pressure waveform via a conformal ultrasonic device. Nat. Biomed. Eng. 2, 687-695 (2018).

11. Bandodkar, A. J. et al. Tattoo-based noninvasive glucose monitoring: a proof-of-concept study. Anal. Chem. https://doi.org/10.1021/ac504300n (2015).

12. Lee, H. et al. Wearable/disposable sweat-based glucose monitoring device with multistage transdermal drug delivery module. Sci. Adv. 3, e1601314 (2017).

13. Kim, J., Campbell, A. S. \& Wang, J. Wearable non-invasive epidermal glucose sensors: a review. Talanta 177, 163-170 (2018).

14. Lee, H., Hong, Y. J., Baik, S., Hyeon, T. \& Kim, D. H. Enzyme-based glucose sensor: from invasive to wearable device. Adv. Healthc. Mater. https://doi. org/10.1002/adhm.201701150 (2018)

15. Kim, J. et al. Simultaneous monitoring of sweat and interstitial fluid using a single wearable biosensor platform. Adv. Sci. https://doi.org/10.1002/ advs.201800880 (2018).

16. Gao, W. et al. Fully integrated wearable sensor arrays for multiplexed in situ perspiration analysis. Nature 529, 509-514 (2016).

17. Imani, S. et al. A wearable chemical-electrophysiological hybrid biosensing system for real-time health and fitness monitoring. Nat. Commun. 7, 11650 (2016).

18. Bruno, G., Anastasova, S. \& Z. Yang, G. A smart wireless ear-worn device for cardiovascular and sweat parameter monitoring during physical exercise: design and performance results. Sensors 19, 1616 (2019).

19. Hong, Y. J. et al. Multifunctional wearable system that integrates sweat-based sensing and vital-sign monitoring to estimate pre-/post-exercise glucose levels. Adv. Funct. Mater. 28, 1805754 (2018).

20. Gomez, H. \& Kellum, J. A. Lactate in sepsis. JAMA 313, 194-195 (2015).

21. Zanella, M. T., Kohlmann, O. \& Ribeiro, A. B. Treatment of obesity hypertension and diabetes syndrome. Hypertension https://doi.org/10.1161/01 hyp.38.3.705 (2001)

22. Cheung, B. M. Y. \& Li, C. Diabetes and hypertension: is there a common metabolic pathway? Curr. Atheroscler. Rep. 14, 160-166 (2012).

23. Epstein, M. \& Sowers, J. R. Diabetes mellitus and hypertension. Hypertension 19, 403-418 (1992).

24. Deedwania, P. Hypertension, dyslipidemia, and insulin resistance in patients with diabetes mellitus or the cardiometabolic syndrome: benefits of vasodilating $\beta$-blockers. J. Clin. Hypertens. 13, 52-59 (2011).

25. Murković, I., Steinberg, M. D. \& Murković, B. Sensors in neonatal monitoring: current practice and future trends. Technol. Health Care 11, 399-412 (2003). 
26. Ho, K. K. Y. et al. Evaluation of an anti-thrombotic continuous lactate and blood pressure monitoring catheter in an in vivo piglet model undergoing open-heart surgery with cardiopulmonary bypass. Chemosensors 8, 56 (2020).

27. Chung, H. U. et al. Binodal, wireless epidermal electronic systems with in-sensor analytics for neonatal intensive care. Science 363, eaau0780 (2019)

28. Yang, J. et al. Prevalence of comorbidities and its effects in patients infected with SARS-CoV-2: a systematic review and meta-analysis. Int. J. Infect. Dis. 94, 91-95 (2020).

29. Sakharov, D. A. et al. Relationship between lactate concentrations in active muscle sweat and whole blood. Bull. Exp. Biol. Med. 150, 83-85 (2010).

30. Halliwill, J. R. Mechanisms and clinical implications of post-exercise hypotension in humans. Exerc. Sport Sci. Rev. 29, 65-70 (2001).

31. Carter, J. R., Stream, S. F., Durocher, J. J. \& Larson, R. A. Influence of acute alcohol ingestion on sympathetic neural responses to orthostatic stress in humans. Am. J. Physiol. Metab. 300, E771-E778 (2011).

32. Roerecke, $M$. et al. The effect of a reduction in alcohol consumption on blood pressure: a systematic review and meta-analysis. Lancet Public Health 2, e108-e120 (2017).

33. Chen, L., Davey Smith, G., Harbord, R. M. \& Lewis, S. J. Alcohol intake and blood pressure: a systematic review implementing a Mendelian randomization approach. PLoS Med. 5, e52 (2008).

34. Maheswaran, R., Gill, J. S., Davies, P. \& Beevers, D. G. High blood pressure due to alcohol. A rapidly reversible effect. Hypertension 17, 787-792 (1991).

35. Ohira, T. et al. Effects of habitual alcohol intake on ambulatory blood pressure, heart rate, and its variability among Japanese men. Hypertension 53, 13-19 (2009).

36. Hauke, A. et al. Complete validation of a continuous and blood-correlated sweat biosensing device with integrated sweat stimulation. Lab Chip 18, 3750-3759 (2018).

37. Monte-Moreno, E. Non-invasive estimate of blood glucose and blood pressure from a photoplethysmograph by means of machine learning techniques. Artif. Intell. Med. 53, 127-138 (2011).

38. Cinar, Y. Blood viscosity and blood pressure: role of temperature and hyperglycemia. Am. J. Hypertens. 14, 433-438 (2001).

39. Filipovsky, J. et al. The relationship of blood pressure with glucose, insulin, heart rate, free fatty acids and plasma cortisol levels according to degree of obesity in middle-aged men. J. Hypertens. 14, 229-235 (1996).

40. Mort, J. R. \& Kruse, H. R. Timing of blood pressure measurement related to caffeine consumption. Ann. Pharmacother. 42, 105-110 (2008).

41. Nurminen, M.-L., Niittynen, L., Korpela, R. \& Vapaatalo, H. Coffee, caffeine and blood pressure: a critical review. Eur. J. Clin. Nutr. 53, 831-839 (1999).

42. Tai, L.-C. et al. Methylxanthine drug monitoring with wearable sweat sensors. Adv. Mater. 30, 1707442 (2018).

43. Ciui, B. et al. Chemical sensing at the robot fingertips: toward automated taste discrimination in food samples. ACS Sens. 3, 2375-2380 (2018).

44. Asano, R. Y. Acute effects of physical exercise in type 2 diabetes: a review. World J. Diabetes 5, 659-665 (2014).

45. Klaric, D. \& Klaric, V. Alcohol-induced coma, hypothermia and hypotension. J. Membr. Sci. Technol. 5, 1000137 (2015).

46. Wilson, E. \& Waring, W. S. Severe hypotension and hypothermia caused by acute ethanol toxicity. Emerg. Med. J. 24, e7 (2007).

47. Hillbom, M., Saloheimo, P. \& Juvela, S. Alcohol consumption, blood pressure, and the risk of stroke. Curr. Hypertens. Rep. 13, 208-213 (2011).

48. Nystoriak, M. A. \& Bhatnagar, A. Cardiovascular effects and benefits of exercise Front. Cardiovasc. Med. https://doi.org/10.3389/fcvm.2018.00135 (2018).
49. Fagard, R. H. Effects of exercise, diet and their combination on blood pressure. J. Hum. Hypertens. 19, S20-S24 (2005).

50. Jia, W. et al. Epidermal biofuel cells: energy harvesting from human perspiration. Angew. Chem. Int. Ed. 52, 7233-7236 (2013).

51. MacDonald, J. R. Potential causes, mechanisms, and implications of post exercise hypotension. J. Hum. Hypertens. 16, 225-236 (2002).

52. Crisafulli, A. et al. Effect of differences in post-exercise lactate accumulation in athletes' haemodynamics. Appl. Physiol. Nutr. Metab. 31, 423-431 (2006)

53. Kokkinos, P. Cardiorespiratory fitness, exercise, and blood pressure. Hypertension 64, 1160-1164 (2014).

54. Ricci, F. \& Palleschi, P. Sensor and biosensor preparation, optimization and applications of Prussian Blue modified electrodes. Biosens. Bioelectron. 21, 389-407 (2005).

55. Zhao, J. et al. A fully integrated and self-powered smartwatch for continuous sweat glucose monitoring. ACS Sens. 4, 1925-1933 (2019).

56. Emaminejad, S. et al. Autonomous sweat extraction and analysis applied to cystic fibrosis and glucose monitoring using a fully integrated wearable platform. Proc. Natl Acad. Sci. USA 114, 4625-4630 (2017).

\section{Acknowledgements}

This research was supported by the UCSD Center of Wearable Sensors (CWS) and National Institutes of Health (grant no. 1R21EB027303-01A1). J.R.S. acknowledges support from $\mathrm{CNPq}$ (grant no. 216981/2014-0). E.D.l.p. acknowledges support from a UC MEXUS-CONACYT collaborative fellowship (2017-2022). A.A.K. acknowledges support from the Fulbright Egyptian Scholar Program (grant no. ay2019-2020). T.S.-a. acknowledges the Royal Golden Jubilee PhD scholarship of the Thailand Research Fund. We thank the Kraton Corporation for providing the SEBS samples.

\section{Author contributions}

J.R.S., M.L. and L.Y. conceived the original project, designed and performed experiments, analysed data, and participated in the figure design and writing of the manuscript. E.D.l.p. performed experiments, analysed data, and participated in the figure design and writing of the manuscript. K.P. and T.S.-a. performed experiments and analysed data. A.N.d.L.S., A.A.K. and F.Z. performed experiments. N.T. fabricated the devices. J.W. and S.X. conceived the original project, designed the experiments, analysed data, participated in the figure design and manuscript writing, and provided guidance to the project.

\section{Competing interests}

The authors declare no competing interests.

\section{Additional information}

Supplementary information The online version contains supplementary material available at https://doi.org/10.1038/s41551-021-00685-1.

Correspondence and requests for materials should be addressed to S.X. or J.W. Reprints and permissions information is available at www.nature.com/reprints. Publisher's note Springer Nature remains neutral with regard to jurisdictional claims in published maps and institutional affiliations.

(c) The Author(s), under exclusive licence to Springer Nature Limited 2021 


\section{Reporting Summary}

Nature Research wishes to improve the reproducibility of the work that we publish. This form provides structure for consistency and transparency in reporting. For further information on Nature Research policies, see our Editorial Policies and the Editorial Policy Checklist.

\section{Statistics}

For all statistical analyses, confirm that the following items are present in the figure legend, table legend, main text, or Methods section.

n/a Confirmed

\The exact sample size $(n)$ for each experimental group/condition, given as a discrete number and unit of measurement

$\bigotimes$ A statement on whether measurements were taken from distinct samples or whether the same sample was measured repeatedly

$\square$ The statistical test(s) used AND whether they are one- or two-sided

$\triangle \square$ Only common tests should be described solely by name; describe more complex techniques in the Methods section.

$\bigotimes$ A description of all covariates tested

$\square$ A description of any assumptions or corrections, such as tests of normality and adjustment for multiple comparisons

$\square$ A full description of the statistical parameters including central tendency (e.g. means) or other basic estimates (e.g. regression coefficient)

$\square \bigotimes \begin{aligned} & \text { A full description of the statistical parameters including central tendency (e.g. means) or other basic estima } \\ & \text { AND variation (e.g. standard deviation) or associated estimates of uncertainty (e.g. confidence intervals) }\end{aligned}$

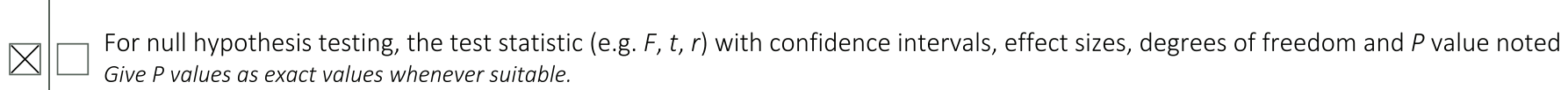

Х $\square$ For Bayesian analysis, information on the choice of priors and Markov chain Monte Carlo settings

Х $\square$ For hierarchical and complex designs, identification of the appropriate level for tests and full reporting of outcomes

Х $\square$ Estimates of effect sizes (e.g. Cohen's $d$, Pearson's $r$ ), indicating how they were calculated

Our web collection on statistics for biologists contains articles on many of the points above.

\section{Software and code}

Policy information about availability of computer code

Data collection Acoustic blood pressure data were captured via an Olympus 507 7PR pulser-receiver, and processed via a custom developed program in LabView (National Instrument, TX). All electrochemical characterization and data acquisition were performed with an electrochemical workstation $\mu$ Autolab III and CHI 1230A. A commercial glucometer (ACCU-CHEK, USA), blood lactate (NOVA biomedical, USA), breathalyzer (BACtrack 580 Pro) and an FDA-approved blood pressure cuff (LOVIA, USA) were used on the subject for obtaining blood glucose, blood lactate, alcohol level, blood pressure and heart rate validation data, respectively.

Data analysis Nova 2.1 software provided by Metrohm and $\mathrm{CHI} 1230 \mathrm{~A}$ software provided by $\mathrm{CH}$ instrument Inc. were used to analyse the acquired electrochemical data. A custom program developed on LabView to convert acoustic signals to a blood-pressure waveform was used to process the acoustic signal in real-time. The blood-pressure waveform data were analysed in Origin Pro 9. 0 to calculate the heart rate.

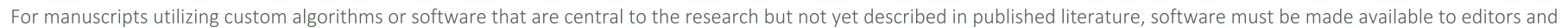

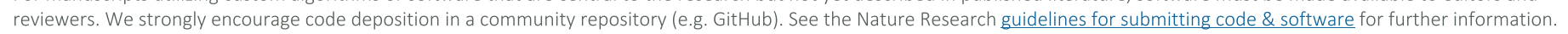

\section{Data}

Policy information about availability of data

All manuscripts must include a data availability statement. This statement should provide the following information, where applicable:

- Accession codes, unique identifiers, or web links for publicly available datasets

- A list of figures that have associated raw data

- A description of any restrictions on data availability

All the data generated and analysed during the study are included in the paper and its supplementary information. 


\section{Field-specific reporting}

Please select the one below that is the best fit for your research. If you are not sure, read the appropriate sections before making your selection.

\Life sciences

Behavioural \& social sciences

Ecological, evolutionary \& environmental sciences

For a reference copy of the document with all sections, see nature.com/documents/nr-reporting-summary-flat.pdf

\section{Life sciences study design}

All studies must disclose on these points even when the disclosure is negative.

Sample size Five consenting subjects, including: one representative subject with a sedentary life style; one representative subject with an active life style; one representative subject caffeine-sensitive (not used to drinking coffee); and two subjects for the alcohol study. All 5 subjects were healthy, and no diabetes, alcoholic or hypertensive patients were recruited.

Data exclusions No data were excluded.

Replication Data acquisition was performed on the different subjects multiple times to verify the functioning of the device.

Randomization The subjects were selected from within the research group, with different self-reported activity levels.

Blinding No blinding measures were taken deliberately, and all data were processed together by multiple authors.

\section{Reporting for specific materials, systems and methods}

We require information from authors about some types of materials, experimental systems and methods used in many studies. Here, indicate whether each material, system or method listed is relevant to your study. If you are not sure if a list item applies to your research, read the appropriate section before selecting a response.

Materials \& experimental systems Methods

$\mathrm{n} / \mathrm{a}$ Involved in the study

\ $\square$ Antibodies $\mathrm{n} / \mathrm{a}$ Involved in the study

Х $\square$ Eukaryotic cell lines

Х $\square$ chIP-seq

\ $\square$ Palaeontology and archaeology

Х $\square$ Flow cytometry

$\bigotimes \square$ Animals and other organisms

Х $\square$ MRI-based neuroimaging

$\square$ \uman research participants

$\bigotimes \square$ Clinical data

$\bigotimes \mid \square$ Dual use research of concern

\section{Human research participants}

Policy information about studies involving human research participants

Population characteristics

Healthy subjects, 20-40 years old.

Recruitment

Consenting subjects were recruited from within the research groups.

Ethics oversight

The institutional review board at the University of California San Diego.

Note that full information on the approval of the study protocol must also be provided in the manuscript. 